\title{
A simplified asymptotic theory for ocean surface electromagnetic wave scattering
}

\author{
A. A. Mouche, B. Chapron \& N. Reul \\ Ifremer, Pointe du Diable, Plouzané, 29280, France \\ *: Corresponding author : Alexis.Mouche@ifremer.fr
}

\begin{abstract}
:
The normalized radar cross-section (NRCS) expression of the Local Curvature Approximation (LCA-1) is derived to first order. The polarization sensitivity of this model is compared to the Kirchhoff Approximation (KA), Two-Scale Model (TSM), Small Slope Approximation (SSA-1) and Small Perturbation Method (SPM-1) to first order in the backscattering configuration. Analytical comparisons and numerical simulations show that LCA-1 and TSM could be rewritten with the same formulation and that their polarization sensitivities are comparable. Comparisons with experimental data acquired in Cand Ku-band reveal that the polarization sensitivities of these models are not adequate. However, the NRCS azimuth modulation predicted by LCA-1 is found to be dependent on polarization and sea surface roughness. This property of the LCA-1 model yields to an azimuth modulation for the polarization ratio. Based on the surface curvature correction concept, a simplified electromagnetic model is proposed. The curvature correction is restricted to the resonant wave-number of the sea roughness spectrum. This is found to reproduce the polarization ratio given by experimental data versus incidence angle and wind speed.
\end{abstract}




\section{1 introduction}

An area of unfilled promised field in ocean remote sensing is the development of a consistent inversion of sea surface geometry and kinematic via the ever-increasing complement of microwave and optical techniques. Since the review by Valenzuela [1], many asymptotic solutions have been proposed to reproduce the observe normalized radar cross-section (NRCS) of the sea surface. Recently, Elfouhaily and Guerin [2] published a very complete review to further advance on this subject. In particular, Elfouhaily pioneered the concept for asymptotic solutions dynamically driven by surface properties, more specifically local curvature characteristics.

However, to date, there are still large discrepancies between observations and predictions. In particular, difficulties exist to fully understand the ratio between co-polarized sea surface NRCS in the backscattering configuration. An advanced model, such as the Small Slope Approximation (SSA) [3], applied to the sea surface has been reported to fail to reproduce this NRCS ratio [4]. Studies based on standard Two-Scale Model (TSM) showed the same limitations [5]. The main issue is the ability to reproduce the NRCS in HH co-polarization. These authors, following others (e.g. [6]), attributed the prediction difference between models and data to difficulties to fully take into account waves with steep slopes which cannot be considered in the framework of these approximations. Other efforts $[4,5,7]$ showed that adding a scalar (non-polarized) 
contribution attributed to wave breaking effects through specular reflection on surface patches with enhanced roughness could help to overcome this issue. However, as the failure of SSA or TSM is observed at any wind speed, solely invoking effect of breaking waves is still a matter of debate. Some authors argue that other surface (bound waves. e.g. [8]) or near-surface processes (sea droplets. e.g. [9]) would yield the same impact on the NRCS.

Our purpose is to use and apply the latest improvements suggested by Elfouhaily and Guerin [2] in the field of the asymptotic electromagnetic modeling to better understand the polarization sensitivity of the scattered signal to the sea surface geometry. Promising studies have already been proposed to use such models in the case of backscattering from an 1-D ocean surface [10]. As already advanced by Elfouhaily and co-authors, the sea surface curvature shall control the polarization sensitivity of the scattered electromagnetic field [11]. In that context, the polarization ratio is dynamically driven by the surface geometry properties. However, questions remain regarding the relative weight of the different sea surface wave scales to such sensitivity. Based on analytical comparisons between models formulations, and comparisons between models prediction and experimental data in the backscattering configuration, we discuss these aspects. A simplified asymptotic solution is then proposed to better understand the expected variability of the sea surface polarization ratio as function of wind speed and direction. The simplified asymptotic theory offers 
tractable statistical solutions, and results are compared to $\mathrm{Ku}-$ and $\mathrm{C}-\mathrm{Band}$ data acquired in VV and $\mathrm{HH}$ polarization configuration.

\section{Position of the problem}

\section{$2.1 \quad$ Coordinates system and definitions}

To expose the general problem in this paper, we adopt the same vectorial conventions than in [2]. The right cartesian coordinate system is defined by the triplet of normalized vectors $(\hat{x}, \hat{y}, \hat{z})$, where the z-axis is directed upward. $\Sigma$ is the rough surface which separates the upper medium and the lower medium (respectively air and water in our specific case). The (sea) surface elevation is represented by $z=\eta(x, y)=\eta(\boldsymbol{r})$, where $\boldsymbol{r}$ is the horizontal component of the three-dimensional position wave vector $\boldsymbol{R}=(\boldsymbol{r}, z)$. According to these conventions, we consider a incident downward propagating electromagnetic plane wave with a wave-vector $\boldsymbol{K}_{0}=\left(\boldsymbol{k}_{0},-q_{0}\right)$. The up-going scattered waves is characterized by the wave-vector $\boldsymbol{K}=\left(\boldsymbol{k}, q_{k}\right) . \boldsymbol{k}_{0}$ and $\boldsymbol{k}$ are the horizontal components of the incident and scattered waves whereas $q_{0}$ and $q_{k}$ are the vertical ones. We define also $\boldsymbol{Q}_{h}$ and $Q_{z}$ related to the coordinates of the wave numbers $\boldsymbol{K}$ and $\boldsymbol{K}_{0}: \boldsymbol{Q}_{h}=\boldsymbol{k}-\boldsymbol{k}_{0}$ and $Q_{z}=q_{0}+q_{k}$.

The scattered field above and far away $(R \rightarrow \infty)$ from the sea surface is 
assumed to be related to the incident wave through the relation:

$$
\boldsymbol{E}_{s}(\vec{R})=-2 i \pi \frac{e^{i K R}}{R} \mathbb{S}\left(\boldsymbol{k}, \boldsymbol{k}_{0}\right) \cdot \hat{E}_{0}
$$

$\mathbb{S}\left(\boldsymbol{k}, \boldsymbol{k}_{0}\right)$ is the so-called scattering operator. $\boldsymbol{E}_{s}(\boldsymbol{R})$ and $\mathbb{S}\left(\boldsymbol{k}, \boldsymbol{k}_{0}\right)$ can be decomposed on the fundamental polarization basis:

$$
\boldsymbol{p}_{v}^{ \pm}( \pm k)=\frac{k \hat{z} \mp q_{k} \hat{k}}{K} \quad \boldsymbol{p}_{h}^{ \pm}( \pm k)=\hat{z} \times \hat{k}
$$

where the subscripts $v$ and $h$ indicate the vertical and horizontal polarizations, respectively. The minus superscript corresponds to the down-going plane waves while the plus superscript to the up-going waves. In this vectors basis, the scattering operator is related to the scattering amplitude $2 \times 2$ matrix through:

$$
\mathbb{S}\left(\boldsymbol{k}, \boldsymbol{k}_{0}\right)=\left[\begin{array}{c}
\boldsymbol{p}_{v}^{-}\left(\boldsymbol{k}_{0}\right) \\
\boldsymbol{p}_{h}^{-}\left(\boldsymbol{k}_{0}\right)
\end{array}\right]^{T} \cdot\left[\begin{array}{c}
\mathbb{S}_{v v}\left(\boldsymbol{k}, \boldsymbol{k}_{0}\right) \mathbb{S}_{v h}\left(\boldsymbol{k}, \boldsymbol{k}_{0}\right) \\
\mathbb{S}_{h v}\left(\boldsymbol{k}, \boldsymbol{k}_{0}\right) \mathbb{S}_{h h}\left(\boldsymbol{k}, \boldsymbol{k}_{0}\right)
\end{array}\right] \cdot\left[\begin{array}{c}
\boldsymbol{p}_{v}^{+}(\boldsymbol{k}) \\
\boldsymbol{p}_{h}^{+}(\boldsymbol{k})
\end{array}\right]
$$

where the superscript $T$ stands for the transpose operator. In the $2 \times 2$ matrix, the first subscript indicates the incident polarization whereas the second one indicates the scattered polarization configuration considered. 
For a given polarization configuration $p q, \mathbb{S}^{p q}\left(\boldsymbol{k}, \boldsymbol{k}_{0}\right)$ is further written as:

$$
\mathbb{S}^{p q}\left(\boldsymbol{k}, \boldsymbol{k}_{0}\right)=\frac{1}{Q_{z}} \int_{\boldsymbol{r}} \mathbb{N}^{p q}\left(\boldsymbol{k}, \boldsymbol{k}_{0} ; \eta(\boldsymbol{r})\right) e^{-i Q_{z} \eta(\boldsymbol{r})} e^{-i \boldsymbol{Q}_{H} \cdot \boldsymbol{r}} d \boldsymbol{r}
$$

where $\mathbb{N}\left(\boldsymbol{k}, \boldsymbol{k}_{0} ; \eta(\boldsymbol{r})\right)$ is a Kernel depending on the approach considered to establish the solution.

The scattering cross-section is given by the incoherent second order statistical expression:

$$
\sigma^{p q}=<\left|\mathbb{S}^{p q}\left(\boldsymbol{k}, \boldsymbol{k}_{0}\right)\right|^{2}>-\left|<\mathbb{S}^{p q}\left(\boldsymbol{k}, \boldsymbol{k}_{0}\right)>\right|^{2}
$$

\subsection{Expansion of the scattering matrix}

Asymptotic electromagnetic models predicting the scattered field from a rough surface can then rely on the expansion of the kernel $\mathbb{N}\left(\boldsymbol{k}, \boldsymbol{k}_{0} ; \eta(\boldsymbol{r})\right)$ in the scattering amplitude expression (4). In particular, Voronovich [3] and Elfouhaily et al. [11] proposed two different expressions for the kernel $\mathbb{N}\left(\boldsymbol{k}, \boldsymbol{k}_{0} ; \eta(\boldsymbol{r})\right)$. These kernels must satisfy known limits, and are defined to reach dynamically the two asymptotic limits of: 
- the SPM-2 solution in the small perturbation limit:

$$
\begin{aligned}
\mathbb{S}\left(\boldsymbol{k}, \boldsymbol{k}_{0}\right) & =\frac{\mathbb{B}\left(\boldsymbol{k}, \boldsymbol{k}_{0}\right)}{Q_{z}} \delta\left(\boldsymbol{Q}_{H}\right)-i \mathbb{B}\left(\boldsymbol{k}, \boldsymbol{k}_{0}\right) \eta\left(\boldsymbol{Q}_{H}\right) \\
& -Q_{z} \int_{\boldsymbol{\xi}} \mathbb{B}_{2}\left(\boldsymbol{k}, \boldsymbol{k}_{0}, \boldsymbol{\xi}\right) \hat{\eta}(\boldsymbol{k}-\boldsymbol{\xi}) \hat{\eta}\left(\boldsymbol{\xi}-\boldsymbol{k}_{0}\right) d \boldsymbol{\xi},
\end{aligned}
$$

where $\mathbb{B}\left(\boldsymbol{k}, \boldsymbol{k}_{0}\right), \mathbb{B}_{2}\left(\boldsymbol{k}, \boldsymbol{k}_{0}, \boldsymbol{\xi}\right)$ are the SPM kernels at first and second order (see e.g. [11]). $\boldsymbol{\xi}$ is the wave number of the sea surface. $\hat{\eta}$ it the Fourier transform of the surface elevation $\eta$.

- the Kirchhoff solution (KA) in the high frequency regime:

$$
\mathbb{S}\left(\boldsymbol{k}, \boldsymbol{k}_{0}\right)=\frac{\mathbb{K}\left(\boldsymbol{k}, \boldsymbol{k}_{0}\right)}{Q_{z}} \int_{\boldsymbol{r}} e^{-i Q_{z} \eta(\boldsymbol{r})} e^{-i \boldsymbol{Q}_{H} \cdot \boldsymbol{r}} d \boldsymbol{r}
$$

where $\mathbb{K}\left(\boldsymbol{k}, \boldsymbol{k}_{0}\right)$ is the Kirchhoff kernel (see e.g. [11]).

A general development of the Kernel $\mathbb{N}\left(\boldsymbol{k}, \boldsymbol{k}_{0}\right)$ can read:

$$
\mathbb{N}\left(\boldsymbol{k}, \boldsymbol{k}_{0} ; \eta(\boldsymbol{r})\right)=\mathbb{N}_{0}\left(\boldsymbol{k}, \boldsymbol{k}_{0}\right)+\mathbb{N}_{1}\left(\boldsymbol{k}, \boldsymbol{k}_{0} ; \eta(\boldsymbol{r})\right)+\mathbb{N}_{2}\left(\boldsymbol{k}, \boldsymbol{k}_{0} ; \eta(\boldsymbol{r})\right)+\ldots
$$


where the sub-kernels up to the second order are expressed as:

$$
\begin{aligned}
& \mathbb{N}_{1}\left(\boldsymbol{k}, \boldsymbol{k}_{0} ; \eta(\boldsymbol{r})\right)=\int_{\boldsymbol{\xi}} N_{1}\left(\boldsymbol{k}, \boldsymbol{k}_{0} ; \boldsymbol{\xi}\right) \hat{\eta}(\boldsymbol{\xi}) e^{i \boldsymbol{\xi} \cdot \boldsymbol{r}} d \boldsymbol{\xi} \\
& \mathbb{N}_{2}\left(\boldsymbol{k}, \boldsymbol{k}_{0} ; \eta(\boldsymbol{r})\right)=\int_{\boldsymbol{\xi}} \int_{\boldsymbol{\xi}^{\prime}} N_{2}\left(\boldsymbol{k}, \boldsymbol{k}_{0} ; \boldsymbol{\xi}, \boldsymbol{\xi}^{\prime}\right) \hat{\eta}(\boldsymbol{\xi}) \hat{\eta}\left(\boldsymbol{\xi}^{\prime}\right) e^{i\left(\boldsymbol{\xi}^{\prime}+\boldsymbol{\xi}\right) \cdot \boldsymbol{r}} d \boldsymbol{\xi} d \boldsymbol{\xi}^{\prime}
\end{aligned}
$$

and are polarization dependent. As discussed by Irisov [12] in the case of the SSA, but also valid for LCA, solutions to satisfy the two asymptotic limits can be easily found. Hereafter, we focus on first order solution (i.e. $\left.\mathbb{N}_{2}\left(\boldsymbol{k}, \boldsymbol{k}_{0}, \boldsymbol{\xi}, \boldsymbol{\xi}^{\prime}\right)=0\right)$ to simply fulfill SPM-1 and KA limits. The SSA-1 (see Eq. (5.12) in [3]) or LCA-1 (see (1) eq in [11]) solutions for the scattering matrix become:

$$
\begin{aligned}
\mathbb{S}\left(\boldsymbol{k}, \boldsymbol{k}_{0}\right) & =\frac{\mathbb{N}_{0}\left(\boldsymbol{k}, \boldsymbol{k}_{0}\right)}{Q_{z}} \int_{\boldsymbol{r}} e^{-i Q_{z} \eta(\boldsymbol{r})} e^{-i \boldsymbol{Q}_{H} \cdot \boldsymbol{r}} d \boldsymbol{r} \\
& -i \int_{\boldsymbol{r}} \int_{\boldsymbol{\xi}} N_{1}\left(\boldsymbol{k}, \boldsymbol{k}_{0} ; \boldsymbol{\xi}\right) \hat{\eta}(\boldsymbol{\xi}) e^{-i Q_{z} \eta(\boldsymbol{r})} e^{-i\left(\boldsymbol{Q}_{H}-\boldsymbol{\xi}\right) \cdot \boldsymbol{r}} d \boldsymbol{\xi} d \boldsymbol{r}
\end{aligned}
$$

with:

$$
\begin{aligned}
\mathbb{N}_{0}\left(\boldsymbol{k}, \boldsymbol{k}_{0}\right) & =\mathbb{B}\left(\boldsymbol{k}, \boldsymbol{k}_{0}\right), \\
N_{1}\left(\boldsymbol{k}, \boldsymbol{k}_{0} ; \boldsymbol{\xi}\right) & =0,
\end{aligned}
$$


for SSA-1 and

$$
\begin{aligned}
\mathbb{N}_{0}\left(\boldsymbol{k}, \boldsymbol{k}_{0}\right) & =\mathbb{K}\left(\boldsymbol{k}, \boldsymbol{k}_{0}\right), \\
N_{1}\left(\boldsymbol{k}, \boldsymbol{k}_{0} ; \boldsymbol{\xi}\right) & =\left[\mathbb{B}\left(\boldsymbol{k}, \boldsymbol{k}_{0} ; \boldsymbol{\xi}\right)-\mathbb{K}\left(\boldsymbol{k}, \boldsymbol{k}_{0}\right)\right],
\end{aligned}
$$

for LCA-1.

\section{$2.3 \quad$ NRCS expression}

Performances of such approaches were demonstrated for LCA (see e.g. [11,13]) and SSA (e.g. [14]), using Monte Carlo Simulations compared with the method of the moments. The statistical average of a large amount of scattered fields using random draws has been found different than analytic expressions of the NRCS directly deduced from the incoherent statistical second order moment. A Monte Carlo scheme applied to a sea surface description is limited by the resolution needed for the small waves and the multi-scale aspect of the sea surface. This aspect directly impacts the results (see discussion in section 3.2). The statistical results presented here are then always compared with measurements to discuss their capabilities. 
The statistical evaluation mostly concerns

$$
<\left|\mathbb{S}^{p q}\left(\boldsymbol{k}, \boldsymbol{k}_{0}\right)\right|^{2}>=<\mathbb{S}^{p q}\left(\boldsymbol{k}, \boldsymbol{k}_{0}\right) \mathbb{S}^{* p q}\left(\boldsymbol{k}, \boldsymbol{k}_{0}\right)>
$$

where the superscript in $X^{*}$ stands for the conjugate quantity of $X$. Up to first order $\mathbb{S}^{p q}\left(\boldsymbol{k}, \boldsymbol{k}_{0}\right)$ and $\mathbb{S}^{* p q}\left(\boldsymbol{k}, \boldsymbol{k}_{0}\right)$ are given by:

$$
\begin{aligned}
\mathbb{S}^{p q}\left(\boldsymbol{k}, \boldsymbol{k}_{0}\right) & =\int_{\boldsymbol{r}}\left[\frac{\mathbb{N}_{0}\left(\boldsymbol{k}, \boldsymbol{k}_{0}\right)}{Q_{z}}-i \tilde{\eta}(\boldsymbol{r})\right] e^{-i Q_{z} \eta(\boldsymbol{r})} e^{-i \boldsymbol{Q}_{H} \cdot \boldsymbol{r}} d \boldsymbol{r} \\
\mathbb{S}^{* p q}\left(\boldsymbol{k}, \boldsymbol{k}_{0}\right) & =\int_{\boldsymbol{r}}\left[\frac{\mathbb{N}_{0}^{*}\left(\boldsymbol{k}, \boldsymbol{k}_{0}\right)}{Q_{z}}+i \tilde{\eta}^{*}\left(\boldsymbol{r}^{\prime}\right)\right] e^{i Q_{z} \eta\left(\boldsymbol{r}^{\prime}\right)} e^{i \boldsymbol{Q}_{H} \cdot \boldsymbol{r}^{\prime}} d \boldsymbol{r}^{\prime},
\end{aligned}
$$

where $\boldsymbol{r}^{\prime}=\boldsymbol{r}+\Delta \boldsymbol{r}$, with $\Delta \boldsymbol{r}$ the correlation length. $\tilde{\eta}(\boldsymbol{r})$ is the modified height function defined as:

$$
\tilde{\eta}(\boldsymbol{r})=\int N_{1}\left(\boldsymbol{k}, \boldsymbol{k}_{0} ; \boldsymbol{\xi}\right) \hat{\eta}(\boldsymbol{\xi}) e^{i \boldsymbol{\xi} \cdot \boldsymbol{r}} d \boldsymbol{\xi}
$$

where $N_{1}\left(\boldsymbol{k}, \boldsymbol{k}_{0} ; \boldsymbol{\xi}\right)$ can be interpreted as a filter of the real height sea surface function $\eta(\boldsymbol{r})$ in the wave number domain. In the case of LCA (see Eq. (15)) and SSA theories, this filter becomes complex for not perfectly conducting surface and are polarization sensitive.

When the first order is neglected, $N_{1}\left(\boldsymbol{k}, \boldsymbol{k}_{0} ; \boldsymbol{\xi}\right)=0$. It follows for the in- 
coherent radar cross-section at zeroth order under Gaussian statistics (see appendix A):

$$
\sigma_{p q}=\left|\frac{\mathbb{N}_{0}\left(\boldsymbol{k}, \boldsymbol{k}_{0}\right)}{Q_{z}}\right|^{2} \int e^{-Q_{z}^{2}[\rho(0)-\rho(\boldsymbol{r})]} e^{-i \boldsymbol{Q}_{H} \cdot \boldsymbol{r}} d \boldsymbol{r},
$$

with $\mathbb{N}_{0}\left(\boldsymbol{k}, \boldsymbol{k}_{0}\right)=\mathbb{B}_{0}\left(\boldsymbol{k}, \boldsymbol{k}_{0}\right)$ and $\mathbb{N}_{0}\left(\boldsymbol{k}, \boldsymbol{k}_{0}\right)=\mathbb{K}_{0}\left(\boldsymbol{k}, \boldsymbol{k}_{0}\right)$, respectively. $\rho(\boldsymbol{r})$, the 2-dimensions correlation function, is defined up to the second harmonic by:

$$
\rho(\boldsymbol{r})=\rho_{0}(\boldsymbol{r})-\rho_{2}(\boldsymbol{r}) \cos 2\left(\phi-\phi_{w}\right),
$$

where $\phi_{w}$ is the wind direction and $\phi$ the scattered azimuth angle. The isotropic part $\rho_{0}(\boldsymbol{r})$ and the anisotropic part $\rho_{2}(\boldsymbol{r})$ are given by:

$$
\begin{aligned}
& \rho_{0}(\boldsymbol{r})=\int_{0}^{\infty} S(k) J_{0}(r k) d k \\
& \rho_{2}(\boldsymbol{r})=\int_{0}^{\infty} S(k) \Delta(k) J_{2}(r k) d k,
\end{aligned}
$$

where $S(k)$ is the omnidirectional elevation spectrum, $\Delta(k)$ the spreading function of the elevation spectrum and $J_{n}$ the $n^{t h}$ order Bessel function of the first kind.

For LCA-1 or SSA-2, the statistical averaging is not as straightforward (see 
appendix A). Indeed, $N_{1}\left(\boldsymbol{k}, \boldsymbol{k}_{0} ; \boldsymbol{\xi}\right) \neq 0$. In the statistical calculation, we neglect the fourth order term as it represents a second order contribution (in $\eta^{2}$ ), whereas the series expansion is limited to the first order (in $\eta$ ). For LCA-1, the following expression for the NRCS is found:

$$
\begin{aligned}
\sigma^{p q}=\frac{1}{Q_{z}^{2}} \int & {\left[\left|\mathbb{K}\left(\boldsymbol{k}, \boldsymbol{k}_{0}\right)\right|^{2}+2 Q_{z}^{2} \operatorname{Re}\left\{\mathbb{K}\left(\boldsymbol{k}, \boldsymbol{k}_{0}\right)[\tilde{\rho}(\Delta \boldsymbol{r})-\tilde{\rho}(0)]\right\}\right] } \\
& e^{-Q_{z}^{2}[\rho(0)-\rho(\Delta \boldsymbol{r})]} e^{-i \boldsymbol{Q}_{H} \cdot \Delta \boldsymbol{r}} d \Delta \boldsymbol{r}
\end{aligned}
$$

We introduced the modified correlation function such as:

$$
\begin{aligned}
<\tilde{\eta}(\boldsymbol{r}) \eta(\boldsymbol{r}+\Delta \boldsymbol{r})> & =\int N_{1}\left(\boldsymbol{k}, \boldsymbol{k}_{0} ; \boldsymbol{\xi}\right) \hat{\eta}(\boldsymbol{\xi}) \hat{\eta^{*}}(\boldsymbol{\xi}) e^{i \boldsymbol{\xi} \cdot \Delta \boldsymbol{r}} d \boldsymbol{\xi} \\
& =\int N_{1}\left(\boldsymbol{k}, \boldsymbol{k}_{0} ; \boldsymbol{\xi}\right) S(\boldsymbol{\xi}) e^{i \boldsymbol{\xi} \cdot \Delta \boldsymbol{r}} d \boldsymbol{\xi}=\tilde{\rho}(\Delta \boldsymbol{r}),
\end{aligned}
$$

where $S(\boldsymbol{\xi})$ is the surface elevation spectrum. To compute $\tilde{\rho}_{0}(\boldsymbol{r})$ and $\tilde{\rho}_{2}(\boldsymbol{r})$, one just have to apply equations (22) and (23) using the filtered omnidirectional spectrum and filtered spreading function. The polarized term exists only if there is a difference between the correlation function of the filtered sea surface in 0 and $\Delta \boldsymbol{r}$. Assuming $N_{1}\left(\boldsymbol{k}, \boldsymbol{k}_{0} ; \boldsymbol{\xi}\right)$ to be at least a quadratic form (i.e. $\left.N_{1}\left(\boldsymbol{k}, \boldsymbol{k}_{0} ; \boldsymbol{\xi}\right) \propto \boldsymbol{\xi}^{\mathbf{2}}\right)$, as already assumed by Elfouhaily et al. [11] or Guerin et al. [15] in the framework of the WCA, the polarization correction shall only results from differences between the second order derivative of the correlation 
function at 0 and $\Delta \boldsymbol{r}$. This only occurs for multi-scale surfaces. At high frequency, $\Delta \boldsymbol{r}$ is small and this difference relates directly to the mean squared surface curvature.

Alternatively, it can be stated that Eq (4) could have been chosen to be expressed using a phase perturbation technique [16]. This method was already applied in [4] for SSA-2. Under this assumption, the general expansion of the scattering matrix up to the second term can be re-written as:

$$
\mathbb{S}\left(\boldsymbol{k}, \boldsymbol{k}_{0}\right)=\mathbb{N}_{0}\left(\boldsymbol{k}, \boldsymbol{k}_{0}\right) \int_{\boldsymbol{r}} e^{-i Q_{z} \Phi\left(\boldsymbol{k}, \boldsymbol{k}_{0} ; \eta(\boldsymbol{r})\right)} e^{-i \boldsymbol{Q}_{H} \cdot \boldsymbol{r}} d \boldsymbol{r},
$$

with:

$$
\Phi\left(\boldsymbol{k}, \boldsymbol{k}_{0} ; \eta(\boldsymbol{r})\right)=\int_{\boldsymbol{\xi}}\left[1+\frac{N_{1}\left(\boldsymbol{k}, \boldsymbol{k}_{0} ; \boldsymbol{\xi}\right)}{\mathbb{N}_{0}\left(\boldsymbol{k}, \boldsymbol{k}_{0}\right)}\right] \hat{\eta}(\boldsymbol{\xi}) e^{i \boldsymbol{\xi} \cdot \boldsymbol{r}} d \boldsymbol{\xi}
$$

where:

$$
N_{1}\left(\boldsymbol{k}, \boldsymbol{k}_{0} ; \boldsymbol{\xi}\right)=\mathbb{B}_{2}\left(\boldsymbol{k}, \boldsymbol{k}_{0} ; \boldsymbol{k}-\boldsymbol{\xi}\right)+\mathbb{B}_{2}\left(\boldsymbol{k}, \boldsymbol{k}_{0} ; \boldsymbol{k}_{0}+\boldsymbol{\xi}\right)+\mathbb{B}\left(\boldsymbol{k}, \boldsymbol{k}_{0}\right),
$$


or

$$
N_{1}\left(\boldsymbol{k}, \boldsymbol{k}_{0} ; \boldsymbol{\xi}\right)=\mathbb{B}\left(\boldsymbol{k}, \boldsymbol{k}_{0} ; \boldsymbol{\xi}\right)-\mathbb{K}\left(\boldsymbol{k}, \boldsymbol{k}_{0}\right),
$$

for SSA-2 and LCA-1, respectively. Applying this development to the SSA and LCA, the NRCS given by SSA-2 and LCA-1 can be written as:

$$
\sigma_{0}(\theta, \phi)=\left|\frac{\mathbb{N}_{0}\left(\boldsymbol{k}, \boldsymbol{k}_{0}\right)}{Q_{z}}\right|^{2} e^{-Q_{z}^{2} \tilde{\rho}(0)} \int_{\boldsymbol{r}}\left[e^{-Q_{z}^{2} \tilde{\rho}(\boldsymbol{r})}-1\right] e^{-i \boldsymbol{Q}_{H} \cdot \boldsymbol{r}} d \boldsymbol{r}
$$

with:

$$
\tilde{\rho}(\boldsymbol{r})=\int_{\boldsymbol{\xi}}\left|1+\frac{N_{1}\left(\boldsymbol{k}, \boldsymbol{k}_{0}, \boldsymbol{\xi}\right)}{\mathbb{N}_{0}\left(\boldsymbol{k}, \boldsymbol{k}_{0}\right)}\right|^{2} S(\boldsymbol{\xi}) e^{i \boldsymbol{\xi} \cdot \boldsymbol{r}} d \boldsymbol{\xi}
$$

where $N_{1}\left(\boldsymbol{k}, \boldsymbol{k}_{0}, \boldsymbol{\xi}\right)$ and $\mathbb{N}_{0}\left(\boldsymbol{k}, \boldsymbol{k}_{0}\right)$ depends on the asymptotic model considered. $\tilde{\rho}(\boldsymbol{r})$ is again the correlation function of a filtered spectrum.

We tested the two computations for the NRCS and the PR predicted by LCA-1 model. The PR is defined as the ratio of the NRCS in VV over HH expressed in linear scale and is presented in $\mathrm{dB}$. This quantity which increases with the incidence angle (see e.g. [17]) is a key parameter to evaluate the polarization performances of models and is also widely used in the remote- 
sensing community for the wind speed fields retrieval using SAR images in HH-polarization (see e.g. [18]). Comparisons between the NRCS and the PR calculated according the two methods are shown on figure 1 . The sea spectrum used is the one proposed by Elfouhaily et al. [20]. We observe that the results are very similar using both computations. In the following we use the phase perturbation technique. The integration over the wave numbers is performed from $2 \pi / 10^{3}$ to $2 \pi / 10^{-3} \mathrm{rad} / \mathrm{m}$ and over the correlation lengths from 0 to $10^{0.1} \mathrm{~m}$. Wave numbers and correlation lengths are defined with a log-scale using polar coordinates. More Details on the numerical implementation can be found in the recent work published by our group [19].

[figure 1 about here]

\subsection{Comparisons between KA, SSA-1 and LCA-1. Analogy with TSM and $S P M-1$}

In this section, we compare KA, LCA-1 and SSA-1 for the NRCS prediction of the sea surface. The 2-dimension sea spectrum used to compute the NRCS is the empirical one proposed by Elfouhaily et al. [20]. We focus on the backscatter configuration. Our concern is the polarization sensitivity as it is an important limitation for most standard models as the SPM-1 or TSM (see appendix B about TSM formulation).

On figure 2, we present the omnidirectional NRCS measured by satellite Ku- 
Band instruments TRMM and NSCAT for a $10 \mathrm{~m} / \mathrm{s}$ wind speed. These NRCS parameters are determined using buoy validations to average measurements for a given wind speed interval. The model results are given by the two zeroth order models SSA-1 and KA in the case of an isotropic sea surface. TRMM NRCS range from 0 to 18 degrees, and from 15 to 55 degrees for NSCAT. SSA-1 is polarization sensitive for incidence angles greater than 0 degree. KA is not polarization sensitive in this configuration for the whole range of incidence angles. The PR for SSA-1 is $10 \mathrm{~dB}$ at 50 degrees whereas KA always gives $0 \mathrm{~dB}$. As frequently used, KA gives satisfactory results to match the data at low incidence angles. For the largest incidence angles, where there is a polarization difference, we observe that none of the two models at is able to match the data. In particular, SSA-1 overestimates the polarization effect. Qualitatively, at larger incidence angles, a correction to SSA-1 should decrease the polarization effect whereas it is the contrary for KA. In the range of incidences where the quasi-specular reflection dominates the backscattering mechanism, KA and SSA-1 are different, and SSA needs the second-order to match Kirchhoff asymptotic solution [22]. As compared to a Geometrical Optics (GO) solution, proportional to a filtered slope distribution, the KA clearly provides much larger results at large incidence angles. The scalar contribution to the NRCS discussed in the context of the TSM [5] can certainly gain to consider KA solutions. 
[figure 2 about here]

On figure 3 (a) and (c), we present NRCS comparisons in VV and $\mathrm{HH}$ polarizations given by KA, SSA-1, LCA-1 and the TSM for a $10 \mathrm{~m} / \mathrm{s}$ wind speed, in the case of an isotropic sea surface in $\mathrm{Ku}-\mathrm{Band}$ and C-Band. KA and SPM-1 are in solid line. To be not confused by the representation, we recall here that in backscatter, KA results are always lower (respectively greater) than those given by SPM-1 in VV (and respectively HH) polarization. SSA-1 is in dotted line, LCA-1 in dashed line, TSM in dashed-dotted line.

[figure 3 about here]

With LCA-1, the polarization effect is less pronounced than with SPM-1 and SSA-1.

As already reported by Voronovich and Zavorotny [4], thanks to comparisons with empirical models for the backscatter in $\mathrm{Ku}$ and C-Band - respectively SASS-II and C-MOD2-I3, SSA-1 polarization effect at high incidence angles are not appropriate to predict of the NRCS of the sea surface. Especially in $\mathrm{HH}$ polarization, for incidence angles larger than $25^{\circ}$, there is a significant underestimation of the NRCS given by SSA-1 (and -2) in comparison with experimental data. Our numerical computations show that SSA-1 tends towards SPM-1 solution too quickly.

The polarization sensitivity of LCA is found smaller. However, according to 
NSCAT measurements (see figure (2)), this polarization effect to correct the KA zeroth order is still too strong (about $8 \mathrm{~dB}$ at $50^{\circ}$ instead of $5.5 \mathrm{~dB}$ ). As obtained, the LCA-1 results are very close to the TSM results. This agreement between LCA-1 and the TSM can be explained analytically. In LCA-1, the zeroth order is exactly the Kirchhoff solution. Then, the first order term provides a curvature correction due to the roughness properties of the surface associated to short scales. Starting from the general form of LCA-1 (Eq. (11) and (14-15)), we can divide the sea surface description in longer and smaller waves as:

$$
\begin{array}{r}
\eta_{L}(\boldsymbol{r})=\int_{\boldsymbol{\xi}=0}^{\boldsymbol{k}_{c}} \hat{\eta}(\boldsymbol{\xi}) e^{i \boldsymbol{\xi} \cdot \boldsymbol{r}} d \boldsymbol{\xi} \\
\eta_{s}(\boldsymbol{r})=\int_{\boldsymbol{\xi}=\boldsymbol{k}_{c}}^{+\infty} \hat{\eta}(\boldsymbol{\xi}) e^{i \boldsymbol{\xi} \cdot \boldsymbol{r}} d \boldsymbol{\xi},
\end{array}
$$

where $k_{c}$ is the cut off wave number such as the curvature contribution for the longer scales $\int_{\boldsymbol{\xi}=0}^{\boldsymbol{k}_{c}} \mathbb{N}_{1}\left(\boldsymbol{k}, \boldsymbol{k}_{0} ; \boldsymbol{\xi}\right) \hat{\eta}(\boldsymbol{\xi}) e^{i \boldsymbol{\xi} \cdot \boldsymbol{r}} d \boldsymbol{\xi}$ is negligible. Following this scale division, LCA-1 writes:

$$
\begin{aligned}
\mathbb{S}\left(\boldsymbol{k}, \boldsymbol{k}_{0}\right) & =\frac{1}{Q_{z}} \int_{\boldsymbol{r}} e^{-i \boldsymbol{Q}_{H} \cdot \boldsymbol{r}} e^{-i Q_{z}\left[\eta_{s}(\boldsymbol{r})+\eta_{L}(\boldsymbol{r})\right]} \\
& \times\left[\mathbb{K}\left(\boldsymbol{k}, \boldsymbol{k}_{0}\right)-i Q_{z} \int_{\boldsymbol{\xi}=\boldsymbol{k}_{c}}^{+\infty} \hat{\eta}_{s}(\boldsymbol{\xi}) \mathbb{N}_{1}\left(\boldsymbol{k}, \boldsymbol{k}_{0} ; \boldsymbol{\xi}\right) e^{i \boldsymbol{\xi} \cdot \boldsymbol{r}} d \boldsymbol{\xi}\right] d \boldsymbol{r},
\end{aligned}
$$

where $\mathbb{N}_{1}\left(\boldsymbol{k}, \boldsymbol{k}_{0} ; \boldsymbol{\xi}\right)=\mathbb{B}\left(\boldsymbol{k}, \boldsymbol{k}_{0} ; \boldsymbol{\xi}\right)-\mathbb{K}\left(\boldsymbol{k}, \boldsymbol{k}_{0}\right)$, which satisfies $\mathbb{N}_{1}\left(\boldsymbol{k}, \boldsymbol{k}_{0} ; \boldsymbol{\xi}\right) \rightarrow 0$ 
when $\boldsymbol{k} \rightarrow 0$. We expand the exponential to first order to obtain:

$$
\begin{aligned}
\mathbb{S}\left(\boldsymbol{k}, \boldsymbol{k}_{0}\right) & =\frac{\mathbb{K}\left(\boldsymbol{k}, \boldsymbol{k}_{0}\right)}{Q_{z}} \int_{\boldsymbol{r}} e^{-i \boldsymbol{Q}_{H} \cdot \boldsymbol{r}} e^{-i Q_{z} \eta_{L}(\boldsymbol{r})} \\
& -i \int_{\boldsymbol{r}} e^{-i Q_{z} \eta_{L}(\boldsymbol{r})} \int_{\boldsymbol{\xi}=\boldsymbol{k}_{c}}^{+\infty} \hat{\eta}_{s}(\boldsymbol{\xi}) \mathbb{B}\left(\boldsymbol{k}, \boldsymbol{k}_{0} ; \boldsymbol{\xi}\right) e^{i \boldsymbol{\xi} \cdot \boldsymbol{r}} d \boldsymbol{\xi} e^{-i \boldsymbol{Q}_{H} \cdot \boldsymbol{r}} d \boldsymbol{r}
\end{aligned}
$$

considering relatively large electromagnetic wave numbers, the first term rapidly decreases away from the specular direction, and the second one simply describes the SPM-1 solution over tilting longer waves. It is exactly the formulation of a two-scale model. As LCA-1 and TSM yield the same results, the small waves which participates to the first order correction terms through a curvature effect cover the same range of wavelength than the tilted small waves of the TSM theory. Unfortunately, numerous studies (see e.g. [5]) demonstrate TSM fails to correctly reproduce the NRCS of the sea surface - especially in HH polarization.

On figure 3 (e) and (d), we present the predicted PR in Ku-Band and C-Band. As demonstrated in previous works $[5,7]$ at 40 degree in C-Band, TSM and SPM-1 overestimate the PR by more than 2 and $3 \mathrm{~dB}$, respectively. Our study shows that the conclusion is the same if we consider LCA-1 or SSA-1 as the numerical simulations results are respectively very close to TSM and SSA-1. In SSA-2 correction, Voronovich takes into account for the non-local second order effect in a local frame and shows that indeed one of 
the effects is to reduce the polarization sensitivity and to reach the Kirchhoff limit in the particular Dirichlet case [22]. But, comparisons with experimental data showed that even this second order effect is not sufficient to reproduce the NRCS over the ocean surface [4]. Consequently, nor SSA or LCA-1 is able to reproduce the observed polarization sensitivity of the NRCS of the sea surface in the backscattering configuration.

As a second step, we present comparisons between KA, SSA-1 and LCA1 versus azimuth angle. The upwind configuration corresponds to 0 degree whereas the downwind and crosswind configurations stand for 180 and 90/270 degree, respectively.

\section{[figure 4 about here]}

On figure 4 (a-d), the NRCS is plotted for VV and HH polarizations versus azimuth angle at 40 degree incidence angle and a $10 \mathrm{~m} / \mathrm{s}$ wind speed in $\mathrm{C}$ and Ku-Band. The representation conventions for the model results are the same than in figure 3. As expected, we observe that the level predicted by the KA is the same in VV and $\mathrm{HH}$ polarization. The azimuth variation is the same for all models with maxima in upwind and downwind configurations, minima in crosswind. More interestingly, the azimuth behavior is clearly polarization sensitive with LCA-1 model. In SSA-1 and KA, for the higher incidence angles where the small perturbation limit is reached, the azimuth modulation comes 
only from the azimuthal spectrum dependency. With LCA-1, this effect is modified by the second term in Eq. (11) which is polarization and surface dependent. This is a fundamental property of LCA-1 approach. Indeed, all data analysis show that the azimuth modulation of the NRCS is polarization dependent $[17,23,24]$. On figures 4(a-d), we also give SPM-1 and TSM. SPM-1 results are very similar to SSA-1, TSM very close to LCA-1 predictions.

On figure 4 (e) and (f), azimuth dependencies of the PR are given. The mean levels are two high in comparisons of radar data (see e.g. [5,7,17]. In particular, on fig 7 (a-c) and 8 for the C-Band in [17] or fig. 3 and 6 for the X-band in [24]), but the use of LCA-1 enables to reproduce an azimuth modulation with the same characteristics as generally revealed by experimental data. As observed previously on figures 2 (a-d), this is due to the second term of LCA-1 which takes into account the azimuth distribution of the small roughness on the sea surface combined with the polarization effects of the curvature correction. If we follow the phase perturbation method approach (see Eq. (30)), the PR is:

$$
P R(\theta, \phi)=\frac{\mathbb{K}_{0}^{V V}\left(\boldsymbol{k}, \boldsymbol{k}_{0}\right) e^{-Q_{z}^{2} \tilde{\rho}^{V V}(0)} \int_{\boldsymbol{r}}\left[e^{-Q_{z}^{2} \tilde{\rho}^{V V}(\boldsymbol{r})}-1\right] e^{-i \boldsymbol{Q}_{H} \cdot \boldsymbol{r}} d \boldsymbol{r}}{\mathbb{K}_{0}^{H H}\left(\boldsymbol{k}, \boldsymbol{k}_{0}\right) e^{-Q_{z}^{2} \tilde{\rho}^{H H}(0)} \int_{\boldsymbol{r}}\left[e^{-Q_{z}^{2} \tilde{\rho}^{H H}(\boldsymbol{r})}-1\right] e^{-i \boldsymbol{Q}_{H} \cdot \boldsymbol{r}} d \boldsymbol{r}},
$$

and PR is surface description, but also polarization dependent thanks to the modified correlation function $\tilde{\rho}^{p p}(\boldsymbol{r})$. As a comparison, the PR given by SSA-1 
or KA models is simply:

$$
P R(\theta, \phi)=\frac{\mathbb{N}_{0}^{V V}\left(\boldsymbol{k}, \boldsymbol{k}_{0}\right)}{\mathbb{N}_{0}^{H H}\left(\boldsymbol{k}, \boldsymbol{k}_{0}\right)},
$$

with $\mathbb{N}_{0}\left(\boldsymbol{k}, \boldsymbol{k}_{0}\right)=\mathbb{B}\left(\boldsymbol{k}, \boldsymbol{k}_{0}\right)$ and $\mathbb{N}_{0}\left(\boldsymbol{k}, \boldsymbol{k}_{0}\right)=\mathbb{K}\left(\boldsymbol{k}, \boldsymbol{k}_{0}\right)$ for SSA-1 and KA. In this expression, information on the sea surface roughness properties is lost.

To summarize, the comparisons presented in this section showed that the polarization sensitivity for SSA-1 and LCA-1 at incidences larger than 30 degrees are too high to reproduce the NRCS of an ocean-like surface and can be associated with the results of SPM-1 and TSM. However, the LCA-1 formalism enables to reproduce an azimuthal behavior for the NRCS in line with observations (see e.g. $[17,24]$ ). We discuss in the next section a first order solution able to better resolve the polarization sensitivity issue of LCA-1 keeping the polarization and surface dependence of the azimuth description of the NRCS of this approach. 


\section{A simplified asymptotic electromagnetic model}

\subsection{Presentation of the model}

Considering the general expansion of the scattering matrix given by Eq. (11) and the conclusions given in the previous part, we simply consider the kernels:

$$
\begin{aligned}
\mathbb{N}_{0}\left(\boldsymbol{k}, \boldsymbol{k}_{0}\right) & =\mathbb{K}_{0}\left(\boldsymbol{k}, \boldsymbol{k}_{0}\right) \\
N_{1}\left(\boldsymbol{k}, \boldsymbol{k}_{0} ; \boldsymbol{\xi}\right) & =-i\left[\mathbb{B}\left(\boldsymbol{k}, \boldsymbol{k}_{0} ; \boldsymbol{\xi}\right)-\mathbb{K}\left(\boldsymbol{k}, \boldsymbol{k}_{0}\right)\right] \delta\left(\boldsymbol{\xi}=\boldsymbol{Q}_{H}\right),
\end{aligned}
$$

where $\delta$ is the dirac function.

By construction, this first order expansion of the scattering matrix fulfills the two conditions given by equations (6) and (7). Indeed, in the small perturbations limit, the expansion of the exponential term in Eq. (11) leads to the SPM-1 solutions. In opposite, when $\boldsymbol{Q}_{H} \rightarrow 0, \mathbb{B}\left(\boldsymbol{k}, \boldsymbol{k}_{0} ; \boldsymbol{\xi}\right)=\mathbb{K}\left(\boldsymbol{k}, \boldsymbol{k}_{0}\right)$. In backscatter, for a given wave number the immediate consequence is that this solution tends toward the KA when the incidence angle decreases. Alternatively, for a given incidence angle, when the incident electromagnetic wavelength tends towards infinity, the high frequency limits is also reached.

In order to establish an analogy with the LCA-1 approach, the second term in LCA which takes into account the small roughnesses effects thanks to the integral over $\boldsymbol{\xi}$, is now solely evaluated for one value of wave number. This 
value corresponds to the Bragg resonant condition: $\boldsymbol{\xi}=\boldsymbol{Q}_{H}$. Thus, our first order term can be seen as a correction to the Kirchhoff solution due to the resonant curvature effect of the sea surface. This model, the Resonant Curvature Approximation (RCA), reduces the polarization correction. A lower polarization sensitivity for RCA than for LCA-1 is indeed simply expected.

To keep the consistency between SSA and LCA expressions, the scattering matrix from RCA writes:

$$
\begin{aligned}
\mathbb{S}\left(\boldsymbol{k}, \boldsymbol{k}_{0}\right)= & \frac{\mathbb{K}\left(\boldsymbol{k}, \boldsymbol{k}_{0}\right)}{Q_{z}} \int_{\boldsymbol{r}} e^{-i Q_{z} \eta(\boldsymbol{r})} e^{-i \boldsymbol{Q}_{H} \cdot \boldsymbol{r}} d \boldsymbol{r} \\
- & i \int_{\boldsymbol{r}} \int_{\boldsymbol{\xi}}\left[\eta(\boldsymbol{\xi})\left[\mathbb{B}\left(\boldsymbol{k}, \boldsymbol{k}_{0} ; \boldsymbol{\xi}\right)-\mathbb{K}\left(\boldsymbol{k}, \boldsymbol{k}_{0}\right)\right] \delta\left(\boldsymbol{\xi}=\boldsymbol{Q}_{H}\right) e^{i \boldsymbol{\xi} \cdot \boldsymbol{r}} d \boldsymbol{\xi}\right] \\
& e^{-i Q_{z} \eta(\boldsymbol{r})} e^{-i \boldsymbol{Q}_{H} \cdot \boldsymbol{r}} d \boldsymbol{r} .
\end{aligned}
$$

This also writes:

$$
\begin{aligned}
\mathbb{S}\left(\boldsymbol{k}, \boldsymbol{k}_{0}\right) & =\frac{\mathbb{K}\left(\boldsymbol{k}, \boldsymbol{k}_{0}\right)}{Q_{z}} \int_{\boldsymbol{r}} e^{-i Q_{z} \eta(\boldsymbol{r})} e^{-i \boldsymbol{Q}_{H} \cdot \boldsymbol{r}} d \boldsymbol{r} \\
& -i\left[\mathbb{B}\left(\boldsymbol{k}, \boldsymbol{k}_{0} ; \boldsymbol{Q}_{H}\right)-\mathbb{K}\left(\boldsymbol{k}, \boldsymbol{k}_{0}\right)\right] \eta\left(\boldsymbol{Q}_{H}\right) \int e^{-i Q_{z} \eta(\boldsymbol{r})} d \boldsymbol{r}
\end{aligned}
$$

and may be compared to the improved Green's function method proposed 
by [25]:

$$
\begin{aligned}
\mathbb{S}\left(\boldsymbol{k}, \boldsymbol{k}_{0}\right) & =\frac{\mathbb{K}\left(\boldsymbol{k}, \boldsymbol{k}_{0}\right)}{Q_{z}} \int_{\boldsymbol{r}} e^{-i Q_{z} \eta(\boldsymbol{r})} e^{-i \boldsymbol{Q}_{H} \cdot \boldsymbol{r}} d \boldsymbol{r} \\
& -i\left[\mathbb{B}\left(\boldsymbol{k}, \boldsymbol{k}_{0} ; \boldsymbol{Q}_{H}\right)-\mathbb{K}\left(\boldsymbol{k}, \boldsymbol{k}_{0}\right)\right] \eta\left(\boldsymbol{Q}_{H}\right) .
\end{aligned}
$$

The difference simply lies in the weighting of the resonant correction term in $\eta\left(\boldsymbol{Q}_{H}\right)$. In particular, as compared to the improved Green's function method, this difference between Eq. (42) and (43) helps to preserve the required shift and tilt invariance properties.

\subsection{Comparison with existing models: $K A, S S A-1$ and LCA-1}

We now compare the performances of the RCA model with SSA-1, LCA-1 and KA. As in the previous section, we start with the backscatter configuration. On figures 5 (a) and (b), we present the NRCS for a $10 \mathrm{~m} / \mathrm{s}$ wind speed versus incidence angle in the upwind configuration for the $\mathrm{Ku}$ and $\mathrm{C}-\mathrm{Band}$. KA results are plotted in solid line. SSA-1 is in dotted line, LCA-1 in dashed line and RCA in dashed-dotted line. As expected, the polarization sensitivity is reduced between RCA and LCA-1. This change in the polarization sensitivity mostly occurs for HH-polarization. Between $\mathrm{C}$ and Ku-Band, we also observe that with RCA, the influence of the polarization correction term added to the Kirchhoff solution decreases for a given incidence angle. This behavior is 
expected as the first order correction is wavelength sensitive - decreasing when the wavelength decreases to match KA in the high frequency limit regime.

\section{[figure 5 about here]}

On figures 5 (c) and (d), we present the PR versus incidence angle for the same models and with the same configuration of wind. The PR given by RCA increases with the incidence angle but is always lower than with SSA-1 and LCA-1, reaching a value of $8 \mathrm{~dB}$ and $7 \mathrm{~dB}$ respectively for Cand $\mathrm{Ku}-\mathrm{Band}$ at $60 \mathrm{deg}$. For an ocean-like sea surface with a $10 \mathrm{~m} / \mathrm{s}$ wind speed, the backscattered power predicted by the RCA model is less polarization sensitive which is the precise issue not resolved with the existing asymptotic electromagnetic models.

Using SSA-1, LCA-1, KA and RCA, we further compare the NRCS and the PR versus azimuth angle. On figure 6 (a-f), the NRCS in both co-polarizations and the $\mathrm{PR}$ are presented versus azimuth angle for a $10 \mathrm{~m} / \mathrm{s}$ wind speed at 40 degrees incidence angle. The representation conventions are the same than for figure 3. Concerning the NRCS, RCA reproduces an azimuth modulation. Looking more precisely, we note, as for LCA-1 and as observed in many experiments $[17,17,24]$, RCA provides an azimuth modulation which depends on the polarization and the electromagnetic wavelength. Thus, the strength of the resonant correction seems sufficient for an ocean-like surface. The two models give different azimuth modulations. They do not come from the sur- 
face description (same sea spectrum as input in the two models) but from the chosen kernels used to establish LCA and RCA.

[figure 6 about here]

Therefore, LCA-1 or RCA are the only models among those presented here able to reproduce an azimuth modulation for the PR.

[figure 7 about here]

Considering these results, a description of the sea surface which would not consider all the surface scales could have consequences on the predicted NRCS and in particular on the PR. To illustrate this, we present on figure 7 (a) and (b), the NRCS in both co-polarizations and the PR as a function of incidence angle in $\mathrm{Ku}-\mathrm{Band}$ given by two different sea surface descriptions. In one case (dashed line for LCA, dashed-dotted line for RCA), we consider the correlation function defined on the wavelength range: $\lambda \in[0.001,1000] \mathrm{m} . \lambda_{0}$ is the incident electromagnetic wavelength and $\lambda$ the sea surface wavelength. In a second one (dashed line with stars for LCA, dashed-dotted line with circles for RCA) the wavelength range of the waves is defined such as $\lambda \in\left[0.001,48 \times \lambda_{0}\right] \mathrm{m}$. This interval is chosen to match the sea surface description used for Monte Carlo simulations by several authors. We observe that the NRCS predicted is completely different using the whole or the high-pass part 
of the wave spectrum. As a first consequence, using a truncated surface yields to a smoother surface description which gives higher non-realistic values for the NRCS near the nadir configuration. In the contrary, at higher incidence angles, the resonant Bragg scattering over dominates. The NRCS predicted tends toward the SSA-1 solution at large incidence angles when the sea surface description is truncated. This is because the weight of the KA part is reduced. Then, on figure 7 (b), the PR predicted by RCA or LCA-1 increases when the largest waves are ignored. If we still consider the backscatter case, this phenomena is explained by the fact that in RCA or LCA, all the waves contribute to the zeroth order of the model through the "non-polarized" Kirchhoff solution (i.e. $\sigma_{\mathrm{KIR}}^{V V}=\sigma_{\mathrm{KIR}}^{H H}$ ), whereas only the small scales provide the first order "polarized" (by opposition to the "non-polarized" Kirchhoff term) correction term. Therefore, in the backscatter case, the PR level is the result of the relative contribution of these "polarized" and "non-polarized" parts. When a part of the longer waves are removed in RCA or LCA, the relative "polarized" contribution increases which leads to the high values of PR. As a consequence, using a Monte-Carlo scheme to predict the NRCS given by RCA or LCA-1 with a too small range of surface scales will lead to an approximative result. 


\subsection{Comparisons with experimental data}

We propose to test the model against data in $\mathrm{Ku}$ and C-Band. To avoid the asymmetry dependency issues strongly affected by the 2-D description of the wave spectrum and by the skewness effects not accounted for here, we focus on the omnidirectional $A_{0}$ parameter of the NRCS. The data-derived scatterometer wind speed models, the NRCS is decomposed in a three terms Fourier model:

$$
\sigma_{0}^{p p}\left(U_{10}, \theta, \phi\right)=A_{0}\left(U_{10}, \theta\right)+A_{1}\left(U_{10}, \theta\right) \cos (\phi)+A_{2}\left(U_{10}, \theta\right) \cos (2 \phi),
$$

where $U_{10}$ is the ten-meters wind speed, $\phi$ the wind direction relative to the radar's azimuth look direction and $\theta$ the radar's incidence angle. In such kind of decomposition, $A_{1}\left(U_{10}, \theta\right)$ and $A_{2}\left(U_{10}, \theta\right)$ allow to reproduce respectively the upwind to downwind and the upwind to crosswind asymmetry. Using a dataset which provides NRCS values in upwind ( $\phi=0 \mathrm{deg})$, crosswind $(\phi=$ 90 or $270 \mathrm{deg})$ and downwind ( $\phi=180 \mathrm{deg})$, we simply deduce $A_{0}\left(U_{10}, \theta\right)$ by combining these NRCS in linear scale:

$$
\begin{aligned}
A_{0}\left(U_{10}, \theta\right)=\frac{1}{4}\left[\sigma_{0}^{p p}\left(U_{10}, \theta, \phi=0^{\circ}\right)+\sigma_{0}^{p p}\left(U_{10}, \theta, \phi=180^{\circ}\right)\right. \\
\left.+\sigma_{0}^{p p}\left(U_{10}, \theta, \phi=270^{\circ}\right)+\sigma_{0}^{p p}\left(U_{10}, \theta, \phi=90^{\circ}\right)\right]
\end{aligned}
$$


[figure 8 about here]

In Ku-Band, we use collocation of NRCS measurement in HH and VV polarizations from NSCAT with the European Center for Medium-Range Weather Forecasts (ECMWF) model surface wind analysis. NSCAT data were collected from may 14, 1997 to June 25, 1997. The surface quality flags within the NSCAT level 1.7 product are used to check the quality of the backscatter coefficients and thus be free from atmospheric, sea ice, or sensor calibration effects. These data were already used by Quilfen et al. [26] study to discuss the up to downwind and up to crosswind asymmetry of the NRCS in Cand $\mathrm{Ku}$-Band. In $\mathrm{C}$-Band, we use the data set in $\mathrm{VV}$ and $\mathrm{HH}$ polarizations obtained with the full-polarimetric STORM radar developed at CETP [17]. These data are collocated with PHAROS buoy $\left(48^{\circ} 31^{\prime} 42 \mathrm{~N}, 5^{\circ} 49^{\prime} 03 \mathrm{~W}\right)$ which provides wind measurements (speed and direction). The data-set was acquired during the VALPARESO experiment. It was carried out by CETP, in collaboration with IFREMER and Météo-France in the context of the ASAR (Advanced Synthetic Aperture Radar) geophysical calibration and validation exercise performed and supported by ESA. It took place from October 19, 2002 to November 21, 2002 off the coast of France and UK (near Atlantic coast of France and English Channel). This instrument and the field experiment are described in details in [17]. We just recall here that the NRCS from STORM after processing are representative of surface areas of about $4 \mathrm{~km} \times 10 \mathrm{~km}$ or 
$5 \mathrm{~km} \times 5 \mathrm{~km}$ depending on the acquisition mode used.

The sea surface spectrum used as input in the surface description is still the unified one [20].

On figure 8 , we present the $\mathrm{PR}$ versus incidence angle for a $10 \mathrm{~m} / \mathrm{s}$ wind speed in C- and ku-Band predicted by the asymptotic electromagnetic models used above and by the STORM and NSCAT DATA. The comparison shows clearly the benefit of the RCA formulation to reproduce the polarization sensitivity induced by the small resonant roughness. Indeed, we observe that RCA leads to a PR level in very close agreement with the data. This agreement is conserved in both VV and $\mathrm{HH}$ polarizations for the two bands.

[figure 9 about here]

On figure 9, we present the PR versus wind speed in C- and $\mathrm{Ku}$-Band. The incidence angle considered here is $37.5 \mathrm{deg}$ in C-Band and 40 deg in $\mathrm{Ku}$ Band. As expected from the previous comparison, the PR mean levels predicted by SSA-1 and LCA-1 are to high. RCA PR level is in very good agreement with the data in both $\mathrm{C}$ - and $\mathrm{Ku}$ - Band for the whole range of wind speed presented here, excepted the light wind speeds below $5 \mathrm{~m} / \mathrm{s}$. This result is really satisfying since no electromagnetic model was able to predict simultaneously the correct trend of the PR versus wind speed and incidence angle. 


\section{4 conclusion}

This paper applies the latest improvements suggested by Elfouhaily and Guerin [2] in the field of the asymptotic modeling. In particular, we present for the first time the NRCS of the sea surface statistically derived using LCA-1 approach [11].

As firstly suggested by Elfouhaily et al. [11], the sea surface curvature controls the polarization sensitivity of the NRCS. Indeed, we show that the PR predicted by LCA-1 was lower than SSA-1 but greater than KA models which do not take into account the sea surface curvature correction. Analytically we demonstrate that the LCA-1 model formulation could be decomposed in two contributions coming from two distinct scales of the sea surface. This yields to a TSM-like formulation where the curvature correction term only applies on the higher frequency sea surface wave spectrum. Numerical tests confirm that in terms of polarization sensitivity, TSM and LCA-1 lead to very close results. Thus, the use of LCA-1 model could help to study the dividing parameter which is implicit and not arbitrary in LCA-1. But, concerning the polarization sensitivity, this study demonstrates that the first order of LCA is not sufficient to reproduce the NRCS from the sea surface.

In the case of a 2-D sea surface description, the LCA-1 approach induces an azimuth modulation dependency of the predicted NRCS which is polarization and surface description dependent as the sea surface curvature correction is 
itself wind direction and polarization dependent. Consequently and in opposite with other existing models presented, LCA-1 predicts an azimuth modulation for the PR. This property of LCA-1 is a major improvement for asymptotic electromagnetic models as several microwave measurements already exhibited this azimuth polarization dependent behavior.

From model comparisons and the failure of SPM-1, TSM, KA, SSA-1 and LCA-1 to reproduce the PR of the sea surface in the microwave domain, we presented a new asymptotic electromagnetic model. The zeroth order term of the model is given by the KA. The first order term is also based on the sea surface curvature correction term but applied only to the resonant scale to limit the correction effect of LCA-1. Accordingly, the RCA expression represents a slight correction to the improved Green's function method [25] that preserves shift and tilt invariance properties. We show that this RCA model predicts a PR in agreement with experimental data versus incidence angle and wind speed. As dynamically surface dependent, RCA allows to predict a PR with an azimuth modulation.

\section{Acknowledgements}

Tanos Tony Elfouhaily, our friend, first student and colleague passed away on July, $26^{\text {th }} 2006$. He was 37 years of age. His inspirational contribution to our present work is obvious and beyond words. We already miss numerous answers 
you proposed.

\section{Appendix A: Statistical averaging}

From Eq. (16-18), we can decompose the scattering matrix product $S^{p q}\left(\boldsymbol{k}, \boldsymbol{k}_{0}\right) S^{* p q}\left(\boldsymbol{k}, \boldsymbol{k}_{0}\right)$ given by a first order asymptotic electromagnetic model relying on an series expansion such as in Eq. (11) into four terms:

$$
\begin{aligned}
<\mathbb{S}^{p q}\left(\boldsymbol{k}, \boldsymbol{k}_{0}\right) \mathbb{S}^{* p q}\left(\boldsymbol{k}, \boldsymbol{k}_{0}\right)> & =<\mathbb{S}^{p q}\left(\boldsymbol{k}, \boldsymbol{k}_{0}\right) S^{* p q}\left(\boldsymbol{k}, \boldsymbol{k}_{0}\right)>\left.\right|_{0} \\
& +<\mathbb{S}^{p q}\left(\boldsymbol{k}, \boldsymbol{k}_{0}\right) S^{* p q}\left(\boldsymbol{k}, \boldsymbol{k}_{0}\right)>\left.\right|_{1} \\
& +<\mathbb{S}^{p q}\left(\boldsymbol{k}, \boldsymbol{k}_{0}\right) S^{* p q}\left(\boldsymbol{k}, \boldsymbol{k}_{0}\right)>\left.\right|_{2} \\
& +<\mathbb{S}^{p q}\left(\boldsymbol{k}, \boldsymbol{k}_{0}\right) S^{* p q}\left(\boldsymbol{k}, \boldsymbol{k}_{0}\right)>\left.\right|_{3},
\end{aligned}
$$


where:

$$
\begin{aligned}
<\mathbb{S}^{p q}\left(\boldsymbol{k}, \boldsymbol{k}_{0}\right) & \mathbb{S}^{* p q}\left(\boldsymbol{k}, \boldsymbol{k}_{0}\right)>\left.\right|_{0}=\left|\frac{\mathbb{N}_{0}\left(\boldsymbol{k}, \boldsymbol{k}_{0}\right)}{Q_{z}}\right|^{2} \\
\times & \int<e^{-i Q_{z}[\eta(\boldsymbol{r})-\eta(\boldsymbol{r}+\Delta \boldsymbol{r})]}>e^{-i \boldsymbol{Q}_{H} \cdot \Delta \boldsymbol{r}} d \Delta \boldsymbol{r} \\
<\mathbb{S}^{p q}\left(\boldsymbol{k}, \boldsymbol{k}_{0}\right) & \mathbb{S}^{* p q}\left(\boldsymbol{k}, \boldsymbol{k}_{0}\right)>\left.\right|_{1}=\frac{\mathbb{N}_{0}\left(\boldsymbol{k}, \boldsymbol{k}_{0}\right)}{Q_{z}} \\
\times & \int<(i) \eta^{*}(\boldsymbol{r}+\Delta \boldsymbol{r}) e^{-i Q_{z}[\eta(\boldsymbol{r})-\eta(\boldsymbol{r}+\Delta \boldsymbol{r})]}>e^{-i \boldsymbol{Q}_{H} \cdot \Delta \boldsymbol{r}} d \Delta \boldsymbol{r} \\
<\mathbb{S}^{p q}\left(\boldsymbol{k}, \boldsymbol{k}_{0}\right) & \mathbb{S}^{* p q}\left(\boldsymbol{k}, \boldsymbol{k}_{0}\right)>\left.\right|_{2}=\frac{\mathbb{N}_{0}^{*}\left(\boldsymbol{k}, \boldsymbol{k}_{0}\right)}{Q_{z}} \\
\times & \int<(-i) \tilde{\eta}(\boldsymbol{r}) e^{-i Q_{z}[\eta(\boldsymbol{r})-\eta(\boldsymbol{r}+\Delta \boldsymbol{r})]}>e^{-i \boldsymbol{Q}_{H} \cdot \Delta \boldsymbol{r}} d \Delta \boldsymbol{r} \\
<\mathbb{S}^{p q}\left(\boldsymbol{k}, \boldsymbol{k}_{0}\right) & \mathbb{S}^{* p q}\left(\boldsymbol{k}, \boldsymbol{k}_{0}\right)>\left.\right|_{3}=\int<\tilde{\eta}(\boldsymbol{r}) \eta^{*}(\boldsymbol{r}+\Delta \boldsymbol{r}) e^{-i Q_{z}[\eta(\boldsymbol{r})-\eta(\boldsymbol{r}+\Delta \boldsymbol{r})]}> \\
\times & e^{-i \boldsymbol{Q}_{H} \cdot \Delta \boldsymbol{r}} d \Delta \boldsymbol{r}
\end{aligned}
$$

When the first order is neglected, $N_{1}\left(\boldsymbol{k}, \boldsymbol{k}_{0} ; \boldsymbol{\xi}\right)=0$. We simply have:

$$
<\mathbb{S}^{p q}\left(\boldsymbol{k}, \boldsymbol{k}_{0}\right) \mathbb{S}^{* p q}\left(\boldsymbol{k}, \boldsymbol{k}_{0}\right)>=<\mathbb{S}^{p q}\left(\boldsymbol{k}, \boldsymbol{k}_{0}\right) \mathbb{S}^{* p q}\left(\boldsymbol{k}, \boldsymbol{k}_{0}\right)>\left.\right|_{0}
$$

Using the fact that the height function $\eta(\boldsymbol{r})$ is an assumed zero mean gaussian variable, $\left\langle e^{\eta}>=e^{<\eta^{2}>/ 2}\right.$. By definition, the correlation function is: $<\eta(\boldsymbol{r}) \eta(\boldsymbol{r}+\Delta \boldsymbol{r})>=\rho(\Delta \boldsymbol{r})$. And, it follows for the incoherent radar cross- 
section at zeroth order:

$$
\sigma_{p q}=\left|\frac{\mathbb{N}_{0}\left(\boldsymbol{k}, \boldsymbol{k}_{0}\right)}{Q_{z}}\right|^{2} \int e^{-Q_{z}^{2}[\rho(0)-\rho(\boldsymbol{r})]} e^{-i \boldsymbol{Q}_{H} \cdot \boldsymbol{r}} d \boldsymbol{r},
$$

For first order expansion solutions, the statistical averaging is not as straightforward. Indeed, $N_{1}\left(\boldsymbol{k}, \boldsymbol{k}_{0} ; \boldsymbol{\xi}\right) \neq 0$. And, we have to consider $<$ $S^{p q}\left(\boldsymbol{k}, \boldsymbol{k}_{0}\right) S^{* p q}\left(\boldsymbol{k}, \boldsymbol{k}_{0}\right)>\left.\right|_{i \in[1,3]}$ in Eq. (A1). Using the statistical relation $<\eta_{1} e^{\eta_{2}}>=<\eta_{1} \eta_{2}>e^{<\eta_{2}^{2}>/ 2}$, we have:

$$
\begin{aligned}
<\mathbb{S}^{p q}\left(\boldsymbol{k}, \boldsymbol{k}_{0}\right) & S^{* p q}\left(\boldsymbol{k}, \boldsymbol{k}_{0}\right)>\left.\right|_{1}=-\mathbb{N}_{0}\left(\boldsymbol{k}, \boldsymbol{k}_{0}\right) \\
\times & \int\left[<\tilde{\eta^{*}}(\boldsymbol{r}+\Delta \boldsymbol{r}) \eta(\boldsymbol{r})>-<\tilde{\eta^{*}}(\boldsymbol{r}+\Delta \boldsymbol{r}) \eta(\boldsymbol{r}+\Delta \boldsymbol{r})>\right](\mathrm{A} 9) \\
\times & e^{-Q_{z}^{2}[\rho(0)-\rho(\Delta \boldsymbol{r})]} e^{-i \boldsymbol{Q}_{H} \cdot \Delta \boldsymbol{r}} d \Delta \boldsymbol{r} \\
<\mathbb{S}^{p q}\left(\boldsymbol{k}, \boldsymbol{k}_{0}\right) \quad & S^{* p q}\left(\boldsymbol{k}, \boldsymbol{k}_{0}\right)>\left.\right|_{2}=\mathbb{N}_{0}^{*}\left(\boldsymbol{k}, \boldsymbol{k}_{0}\right) \\
\times & \int[<\tilde{\eta}(\boldsymbol{r}) \eta(\boldsymbol{r})>-<\tilde{\eta}(\boldsymbol{r}) \eta(\boldsymbol{r}+\Delta \boldsymbol{r})>] \\
& \times e^{-Q_{z}^{2}[\rho(0)-\rho(\Delta \boldsymbol{r})]} e^{-i \boldsymbol{Q}_{H} \cdot \Delta \boldsymbol{r}} d \Delta \boldsymbol{r}
\end{aligned}
$$

We neglect the fourth order term $<\mathbb{S}^{p q}\left(\boldsymbol{k}, \boldsymbol{k}_{0}\right) S^{* p q}\left(\boldsymbol{k}, \boldsymbol{k}_{0}\right)>\left.\right|_{4}$. Indeed, it represents a second order contribution (in $\eta^{2}$ ), whereas we limit the series expansion to the first order (in $\eta$ ). Using Eq. (A8), (A9) and (A10) and LCA kernel basic properties of reciprocity (see Eq. (6) in [11]), the expression for 
the NRCS is :

$$
\begin{aligned}
\sigma^{p q} & =\frac{1}{Q_{z}^{2}} \int\left[\left|\mathbb{N}_{0}\left(\boldsymbol{k}, \boldsymbol{k}_{0}\right)\right|^{2}+2 Q_{z}^{2} \operatorname{Re}\left\{\mathbb{N}_{0}\left(\boldsymbol{k}, \boldsymbol{k}_{0}\right)[\tilde{\rho}(\Delta \boldsymbol{r})-\tilde{\rho}(0)]\right\}\right] \\
& \times e^{-Q_{z}^{2}[\rho(0)-\rho(\Delta \boldsymbol{r})]} e^{-i \boldsymbol{Q}_{H} \cdot \Delta \boldsymbol{r}} d \Delta \boldsymbol{r}
\end{aligned}
$$

\section{Appendix B: Two-Scale Model expression}

The expression of the NRCS given for the TSM [1]:

$$
\sigma_{0}(\theta, \phi)=\int_{-\infty}^{\infty} d(\tan \Psi) \int_{-\infty}^{\infty} d(\tan \delta) \sigma_{0}^{b r}\left(\theta_{i}\right) P(\tan \Psi, \tan \delta)
$$

where $P(\tan \Psi, \tan \delta)$ is the joint probability density of slopes for the long waves, $\theta_{i}$ the local angle, and $\sigma_{0}^{b r}$ the NRCS given by the SPM- 1 due to the small roughness elements modulated by the longer waves. In our calculation this probability density is assumed Gaussian. The calculation of $\sigma_{0}^{b r}$ is done considering the angles corrections given by Elfouhaily et al. [21] instead of initial Valenzuela's results [1]:

$$
\theta_{i}=-\cos ^{-1}\left[\cos (\theta+\Psi) \cos \left(\tan ^{-1} \delta \cos \Psi\right)\right]
$$

with $S_{x}=\tan \Psi \quad$ and $\quad S_{y}=\tan \delta$. 


\section{References}

[1] Valenzuela, G.R., 1978, Theory for the interaction of electromagnetic waves and oceanic waves. A review. Radio Sciences, 13, 61-85.

[2] Elfouhaily, T. and Guerin, C.-A., 2004, A critical survey of approximate wave theories from random rough surfaces. Waves in Random Media, 14, R1-R40.

[3] Voronovich AG, 1994, Small Slope Approximation for electromagnetic wave at a rough interface of two dielectric half-spaces, Waves in Random Media, 4, 337-367.

[4] Voronovich, A.G. and Zavorotny, V.U., 2001, Theoritical model for scattering of radar signals in $\mathrm{Ku}$ and $\mathrm{C}$ bands from a rough sea surface with breaking waves. Waves in Random Media, 11, $247-269$.

[5] Kudryavtsev, V.N., Hauser, D., Caudal, G. and Chapron, B., 2003, A semi-empirical model of the normalized radar cross section of the sea surface: 1. Background model. Journal of Geophysical Research, 108(C), 8054, doi:10.1029/2001JC001003.

[6] Phillips O.M., 1988, Radar return from the sea surface - Bragg scattering and breaking waves Journal of Fluid Mechanics, 156, 505-531.

[7] Mouche, A.A., Hauser D. and Kudryavtsev, V.N., 2006, Radar scattering of the ocean surface and sea-roughness properties: A combined analysis from dual-polarizations airborne radar observations and models in C band. Journal of Geophysical Research, 111, C09004, doi:10.1029/2005JC003166.

[8] Plant, W.J., Keller W.C., Hesany V., Hara T., Block E. and Donelan, M., 1999, Bound waves and Bragg scattering in wind-wave tank. Journal of Geophysical Research, 104(C2), 3243-3264, doi:10.1029/1998JC900061.

[9] Plant, W.J., 2003, Microwave sea return at moderate to high incidence angles. Waves in Random Media, 13(4), 339-354.

[10] Bourlier, C., Déchamps, N. and Berginc G., 2005, Comparison of Asymptotic Backscattering Models (SSA, WCA, LCA) from one-dimensional Gaussian ocean-like surfaces. IEEE Transaction on Antennas and Propagation, 53(5), 1640-1652, doi:10.1109/TAP.2005.846800.

[11] Elfouhaily, T., Guignard, S., Awadallah, R. and Thompson, D. R., 2003, Local and non-local curvature approximation: A new asymptotic theory for wave scattering. Waves in Random Media, 13(4), 321-337. 
[12] Irisov, V.G., 1994, Small-slope expansion for electromagnetic-wave diffraction on a rough surface. Waves in Random Media, 4, 441-452.

[13] Elfouhaily, T. and Johnson, J. T., 2006, Extension of the local curvature approximation to third order and full tilt invariance, Waves in Random and Complex Media, 16(2), 97-119.

[14] Soriano G., Guerin, C.-A. and Saillard, M., 2002, Scattering by two-dimensional rough surfaces: Comparison between the method of moments, Kirchhoff and small-slope approximations, Waves in Random Media, 12(1), 63-83.

[15] Guerin, C.-A., Soriano, G. and Elfouhaily, T., 2004, Weighted curvature approximation: numerical tests for 2D dielectric surfaces, Waves in Random Media, 14(3), 349-363.

[16] Berman, D.H. and Dacol, D.K., 1990, Manifestly reciprocal scattering amplitudes for rough surfaces interface scattering, Journal of the Acoustical Society of America, 87(5), 2024-2032.

[17] Mouche, A.A., Hauser, D., Daloze, J.-F. and Guerin, C., 2005, Dual-polarization measurements at $\mathrm{C}$ band over the ocean: Results from airborne radar observations and comparison with ENVISAT ASAR data. IEEE Transaction on Geoscience and Remote Sensing, 43(4), 753-769, doi:10.1109/TGRS.2005.843951.

[18] Thompson, D.R., Elfouhaily, T. and Chapron, B., 1998, Polarization ratio for microwave backscattering from ocean surface at low to moderate incidence angles, Paper presented at the International Geoscience and Remote Sensing Symposium, in USA, Seattle, WA.

[19] Reul, N., Tenerelli, J., Chapron, B. and Waldteufel, P, 2006. Modeling Sun glitter at L-band for the sea surface salinity remote sensing with SMOS. accepted for publication in IEEE Transaction on Geoscience and Remote Sensing, MicroRad06 Special issue paper

[20] Elfouhaily, T., Chapron, B., Katsaros, K. and Vandemark, D., 1997, A unified directional spectrum for long and short wind driven waves, Journal of Geophysical Research, 102(C7), 1578115796, doi:10.1029/97JC00467.

[21] Elfouhaily, T., Thompson, D.R., Vandemark, D. and Chapron, B., 1999, A new bistatic model for electromagnetic scattering from perfect conducting random surfaces, Waves in Random Media, 9, 281-294.

[22] Voronovich A.G., 1994, Waves scattering from rough surfaces, Springer Series on Wave Phenomena, Springer.

[23] Unal, C.M.H., Snoeij, P. and Swart, P.J.F., 1991, The polarization-dependent relation between 
radar backscatter from the ocean surface and surface wind vector at frequencies between 1 and 18 GHz. IEEE Transaction on Geoscience and Remote Sensing, 43(4), 621-627.

[24] Hauser, D., Dubois, P., Caudal, C., 1997, Polarimetric wind-scatterometer measurements during POLRAD'96, Paper presented at the International workshop POLRAD'96, The Netherlands, in Noordwijk, ESTEC, 55-64.

[25] Shaw, W.T. and Dougan, A.J., 1998, Green's function refinement as an approach to radar backscatter: General theory and application to LGA scattering from the ocean, IEEE Transaction on Antenna Propagation, 46(1), 57-66.

[26] Quilfen, Y., Chapron, B., Bentamy, A., Gourrion, J., Elfouhaily, T., and Vandemark, D., 1999, Global ERS-1/2 and NSCAT observations: upwind/crosswind and upwind/downwind measurements, Journal of Geophysical Research, 104(C5), 11459-11470, doi:10.1029/1998JC900113.

\section{Figures captions}

Figure 1: Left: NRCS versus incidence angle in $\mathrm{HH}$ and VV polarization. VV signal is larger than HH. Right: PR versus incidence angle. Results were obtained for a $10 \mathrm{~m} / \mathrm{s}$ wind speed in the case of an isotropic surface at $\mathrm{Ku}$-Band and are predicted using LCA-1 using the two methods (see Eq. (31) and Eq. (24) proposed for the NRCS expression.

Figure 2: Left: NRCS versus incidence angle in $\mathrm{HH}$ and $\mathrm{VV}$ polarization. VV signal is larger than HH. Right panel: PR versus incidence angle. Results of models were obtained for a $10 \mathrm{~m} / \mathrm{s}$ wind speed in the case of an isotropic surface at Ku-Band using KA and SSA-1. Date are from TRMM and NSCAT sensors. 
Figure 3: Top panel: NRCS versus incidence angle in $\mathrm{HH}$ and VV polarization for a $10 \mathrm{~m} / \mathrm{s}$ wind speed in the case of an isotropic surface. VV signal is larger than HH. Bottom panel: PR versus incidence angle for a $10 \mathrm{~m} / \mathrm{s}$ wind speed in the case of an isotropic surface. Results are in in C-Band (left) and Ku-Band (right) and are predicted using SPM-1, KA, TSM, SSA-1 and LCA-1.

Figure 4: Top panel: NRCS versus azimuth angle in VV polarization for a $10 \mathrm{~m} / \mathrm{s}$ wind speed and a 40 degrees incidence angle. Middle panel: same but for HH-polarization. Bottom panel: same but for the polarization ratio. Results are in in $\mathrm{C}$-Band (left) and $\mathrm{Ku}$-Band (right) and are predicted using KA, SSA-1, LCA-1, TSM and SPM-1(or Bragg) (see legend).

Figure 5: Top panel: NRCS versus incidence angle in $\mathrm{HH}$ and $\mathrm{VV}$ polarization for a $10 \mathrm{~m} / \mathrm{s}$ wind speed in the upwind configuration. VV signal is larger than HH. Bottom panel: $\mathrm{PR}$ versus incidence angle for a $10 \mathrm{~m} / \mathrm{s}$ wind speed in the upwind configuration. Results are in C-Band (left) and Ku-Band (right) and are predicted using SPM-1, KA, TSM, SSA-1 and LCA-1.

Figure 6: Top panel: NRCS versus azimuth angle in VV polarization 
for a $10 \mathrm{~m} / \mathrm{s}$ wind speed and a 40 degrees incidence angle. Middle panel: same but for HH-polarization. Bottom panel: same but for the polarization ratio. Results are in $\mathrm{C}-\mathrm{Band}$ (left) and $\mathrm{Ku}-\mathrm{Band}$ (right) and are predicted using KA, SSA-1, LCA-1, TSM and SPM-1 (see legend).

Figure 7: Left panel: NRCS versus incidence angle in VV polarization for a $10 \mathrm{~m} / \mathrm{s}$ wind speed in the case of an isotropic surface. Middle panel: Same in HH polarization. Right panel: PR versus incidence angle for a $10 \mathrm{~m} / \mathrm{s}$ wind speed in the case of an isotropic surface. Results are in Ku-Band and are predicted using RCA and LCA-1 coupled with a truncated (larger waves than $48 \times \lambda_{0}$ are removed) and a complete wave spectrum.

Figure 8: $\mathrm{PR}$ versus incidence angle for a $10 \mathrm{~m} / \mathrm{s}$ wind speed in the case of an isotropic surface. Results are in C-Band (left) and Ku-Band (right) and are predicted using KA, SSA-1, LCA-1 and RCA. C-Band data are from STORM radar. Ku-Band data are from NSCAT data.

Figure 9: PR versus wind speed in the case of an isotropic surface. Results are in C-Band for a $37.5 \mathrm{deg}$ incidence angle (left) and $\mathrm{Ku}$-Band for a $40 \mathrm{deg}$ incidence angle (right) and are predicted using SPM-1, KA, TSM, SSA-1 and LCA-1. C-Band data are from STORM radar. Ku-Band data are 
from NSCAT data. 
Waves in Random and Complex Media:

A Simplified Asymptotic Theory for Ocean Surface

Electromagnetic Wave Scattering.

A. A. Mouche, B. Chapron \& N. Reul,

Ifremer, Pointe du Diable, Plouzané, 29280, France

(v1.0 released and submitted September 2006)

Figures

Waves in Random and Complex Media ISSN 1745-5030 print/ ISSN 1745-5049 online (C)2005 Taylor \& Francis Ltd http://www.tandf.co.uk/journals

DOI: $10.1080 / 17455030 \mathrm{xxxxxxxxxxxxx}$ 
(a)

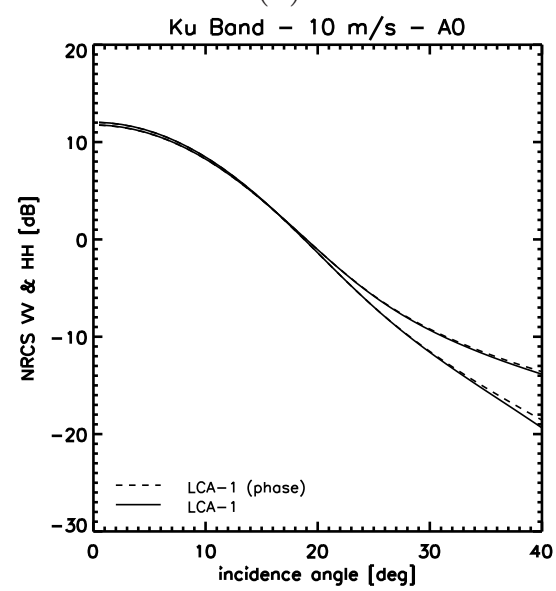

(b)

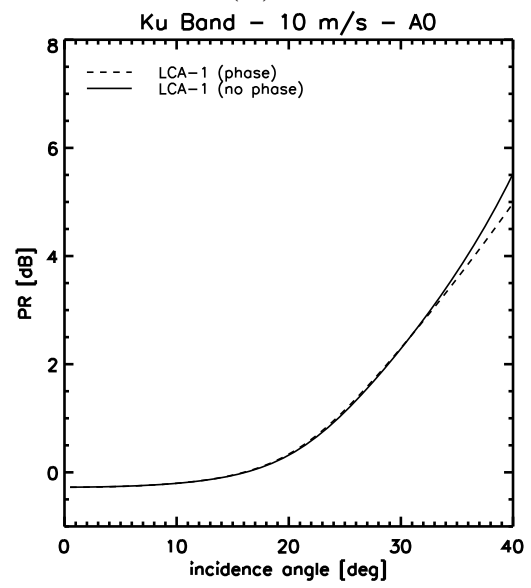

Figure 1. Left: NRCS versus incidence angle in HH and VV polarization. VV signal is larger than HH. Right: PR versus incidence angle. Results were obtained for a $10 \mathrm{~m} / \mathrm{s}$ wind speed in the case of an isotropic surface at $\mathrm{Ku}$-Band and are predicted using LCA-1 using the two methods (see Eq. (31) and Eq. (24) proposed for the NRCS expression.

(a)

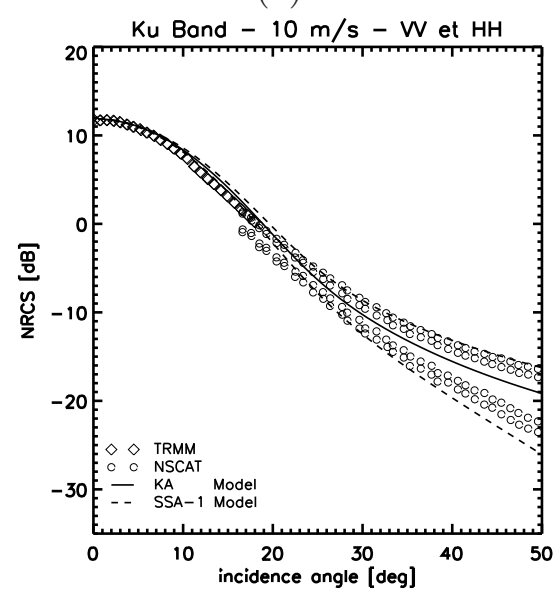

(b)

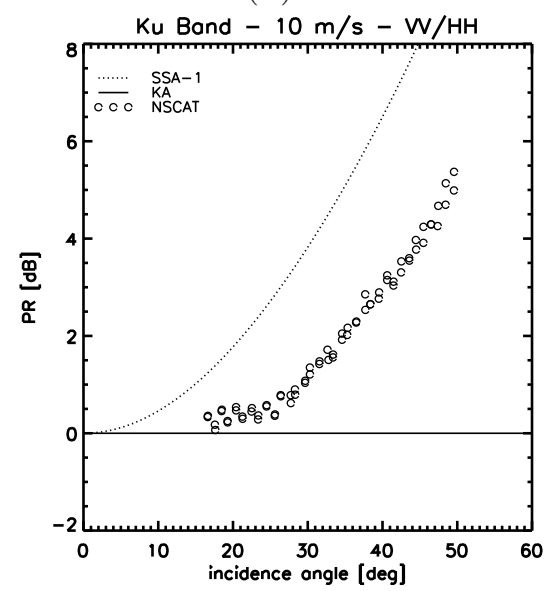

Figure 2. Left: NRCS versus incidence angle in HH and VV polarization. VV signal is larger than HH. Right panel: PR versus incidence angle. Results of models were obtained for a $10 \mathrm{~m} / \mathrm{s}$ wind speed in the case of an isotropic surface at Ku-Band using KA and SSA-1. Date are from TRMM and NSCAT sensors. 
(a)

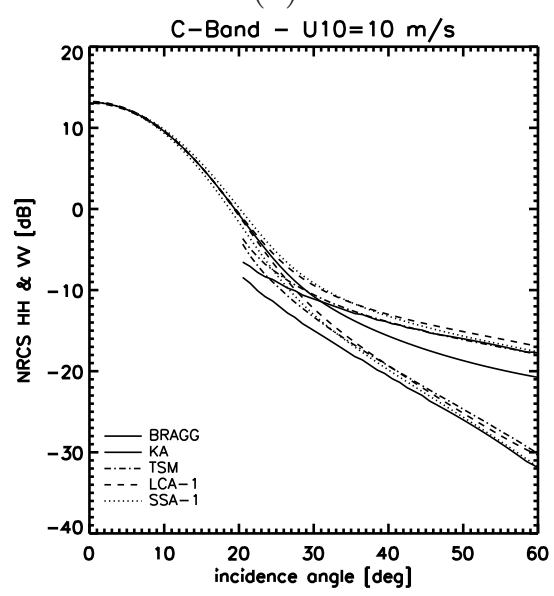

(c)

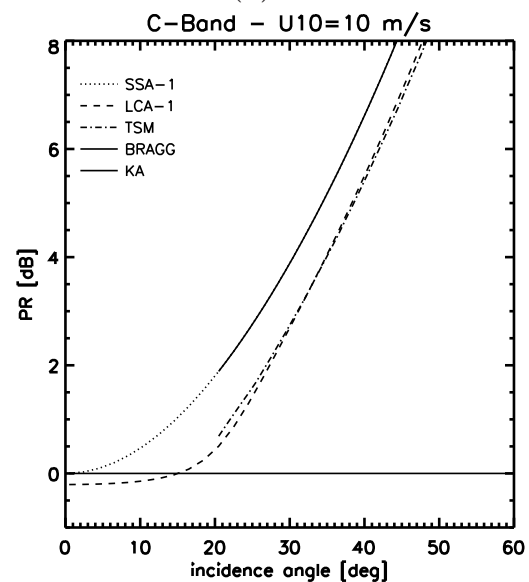

(b)

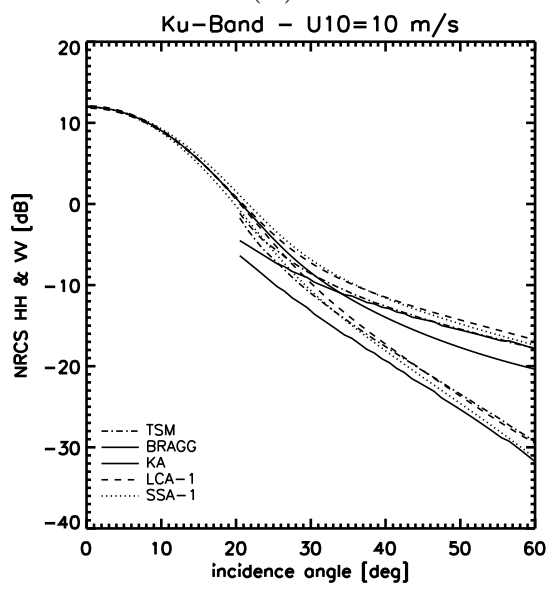

(d)

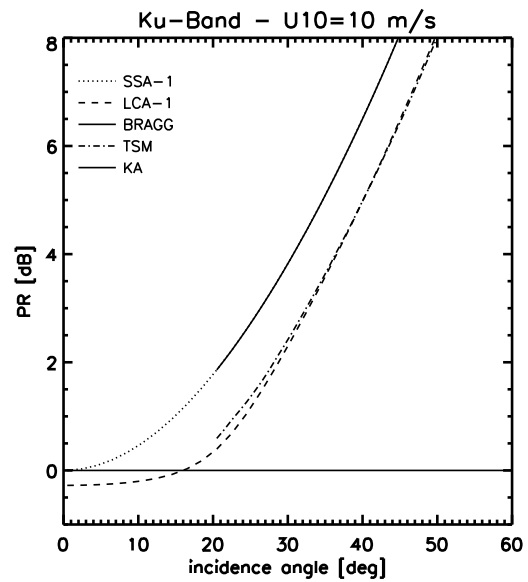

Figure 3. Top panel: NRCS versus incidence angle in HH and VV polarization for a $10 \mathrm{~m} / \mathrm{s}$ wind speed in the case of an isotropic surface. VV signal is larger than HH. Bottom panel: PR versus incidence angle for a $10 \mathrm{~m} / \mathrm{s}$ wind speed in the case of an isotropic surface. Results are in in C-Band (left) and Ku-Band (right) and are predicted using SPM-1, KA, TSM, SSA-1 and LCA-1. 
(a)

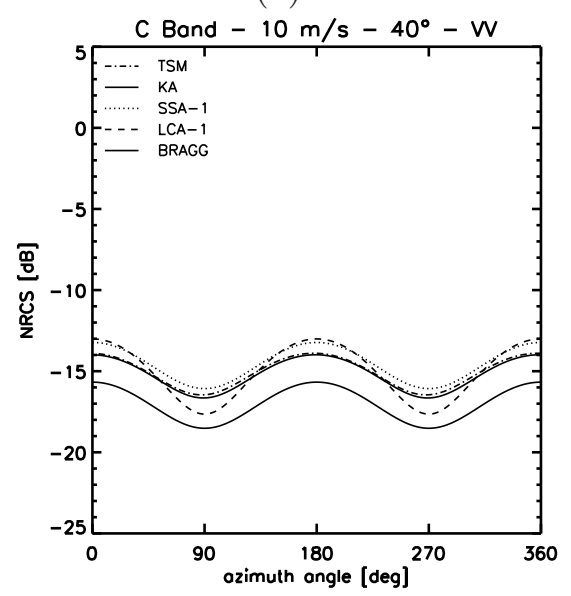

(c)

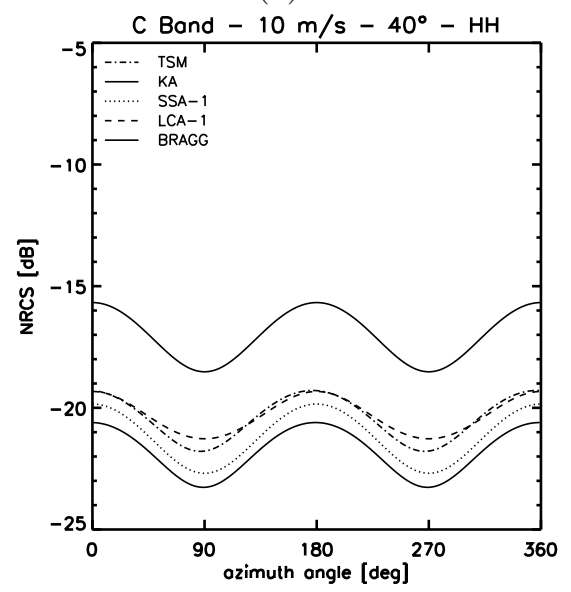

(e)

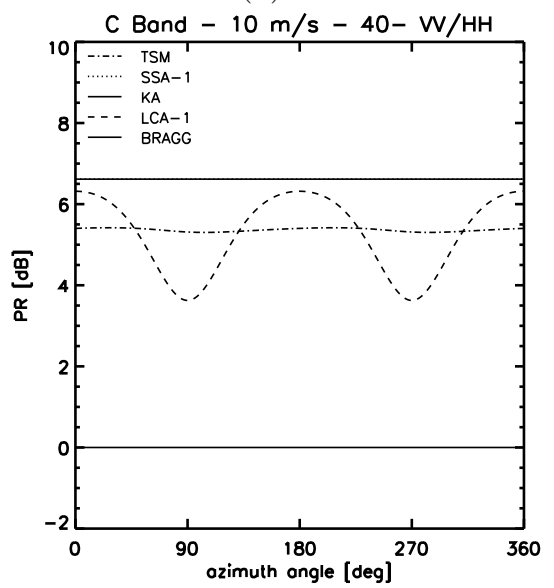

(b)

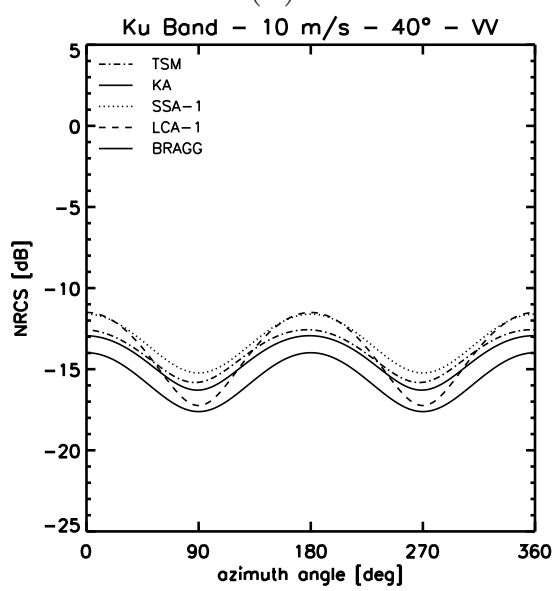

(d)

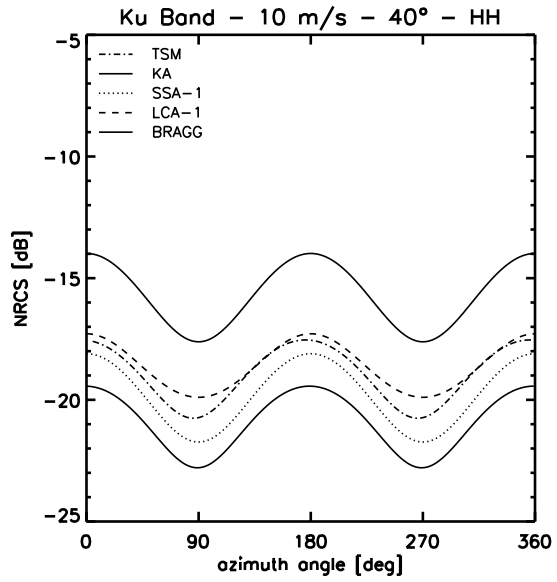

(f)

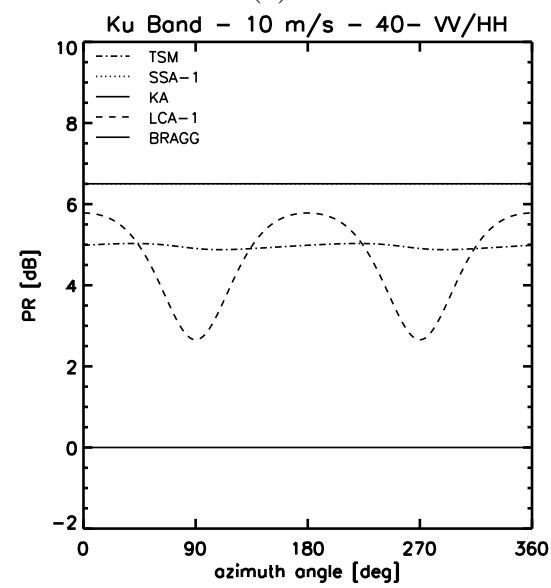

Figure 4. Top panel: NRCS versus azimuth angle in VV polarization for a $10 \mathrm{~m} / \mathrm{s}$ wind speed and a 40 degrees incidence angle. Middle panel: same but for $\mathrm{HH}$-polarization. Bottom panel: same but for the polarization ratio. Results are in in $\mathrm{C}$-Band (left) and $\mathrm{Ku}-\mathrm{Band}$ (right) and are predicted using KA, SSA-1, LCA-1, TSM and SPM-1(or Bragg) (see legend). 
(a)

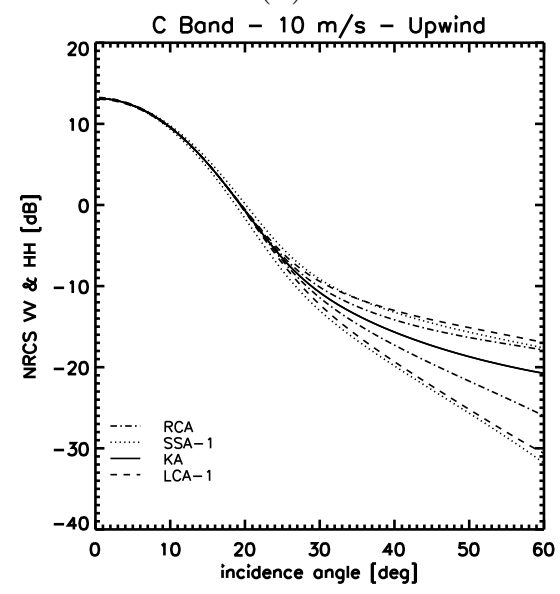

(c)

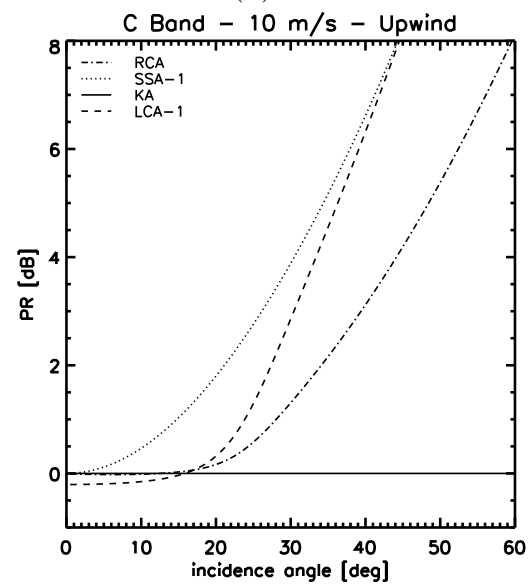

(b)

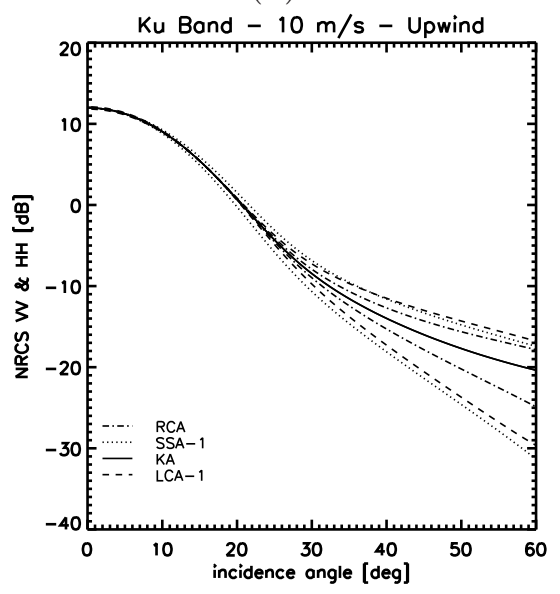

(d)

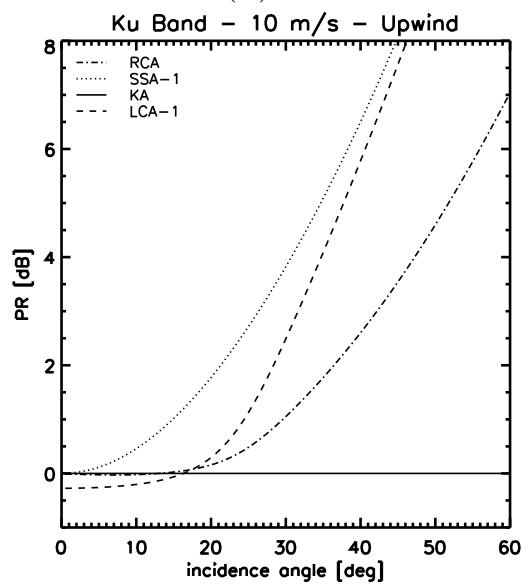

Figure 5. Top panel: NRCS versus incidence angle in $\mathrm{HH}$ and VV polarization for a $10 \mathrm{~m} / \mathrm{s}$ wind speed in the upwind configuration. VV signal is larger than HH. Bottom panel: PR versus incidence angle for a $10 \mathrm{~m} / \mathrm{s}$ wind speed in the upwind configuration. Results are in C-Band (left) and Ku-Band (right) and are predicted using SPM-1, KA, TSM, SSA-1 and LCA-1. 
(a)

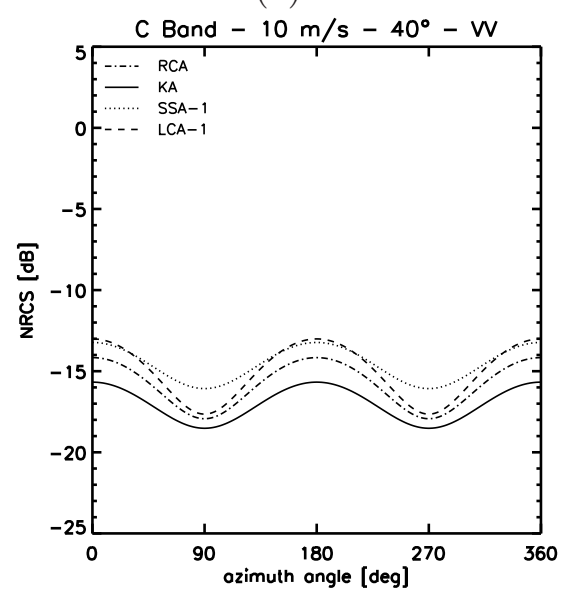

(c)

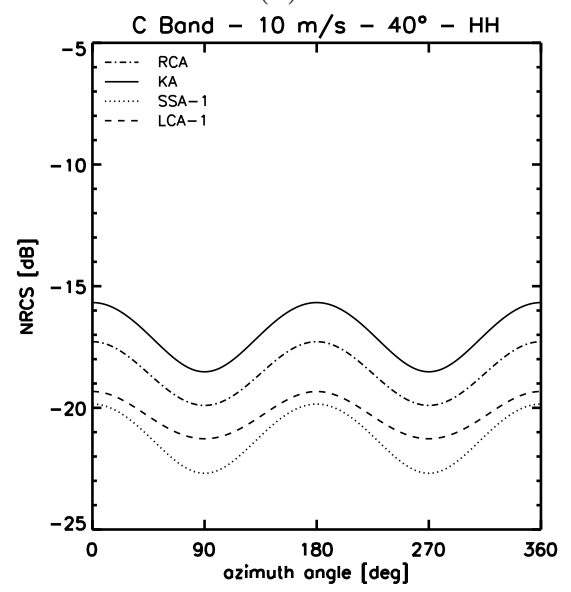

(e)

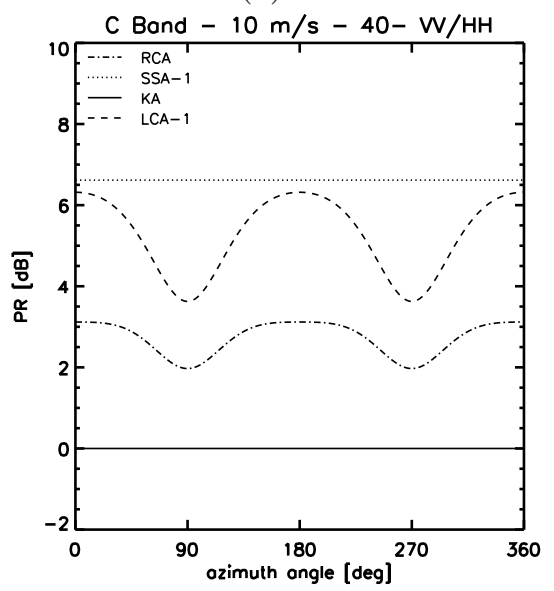

(b)

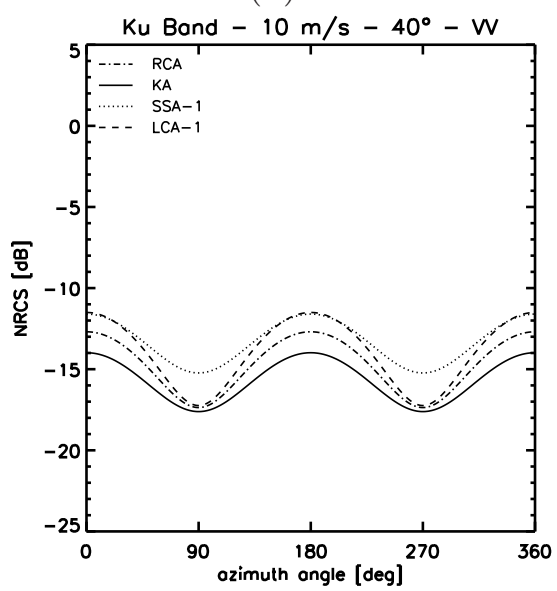

(d)

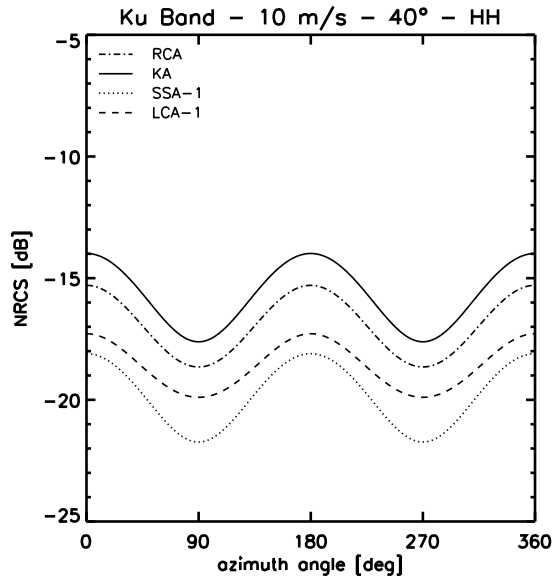

(f)

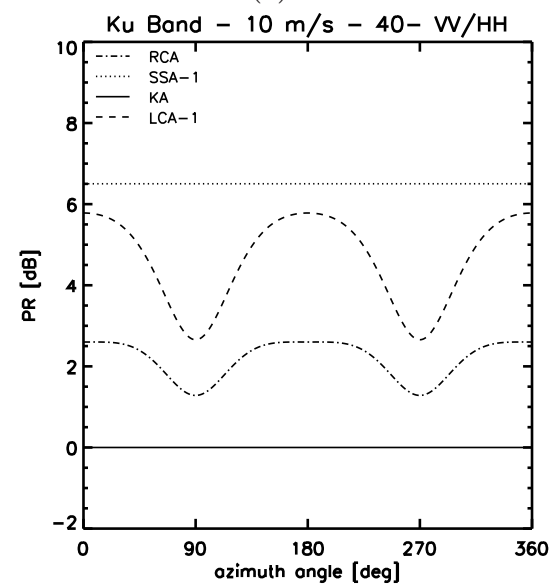

Figure 6. Top panel: NRCS versus azimuth angle in VV polarization for a $10 \mathrm{~m} / \mathrm{s}$ wind speed and a 40 degrees incidence angle. Middle panel: same but for HH-polarization. Bottom panel: same but for the polarization ratio. Results are in $\mathrm{C}$-Band (left) and $\mathrm{Ku}$-Band (right) and are predicted using KA, SSA-1, LCA-1, TSM and SPM-1 (see legend). 
(a)

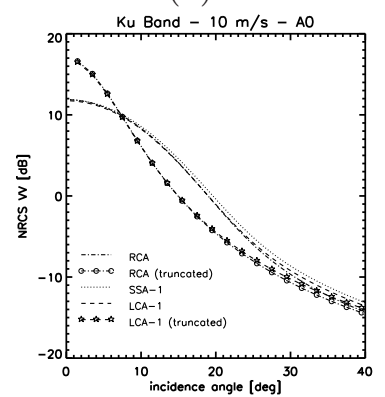

(b)

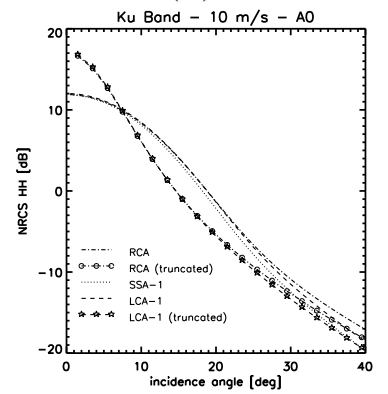

(c)

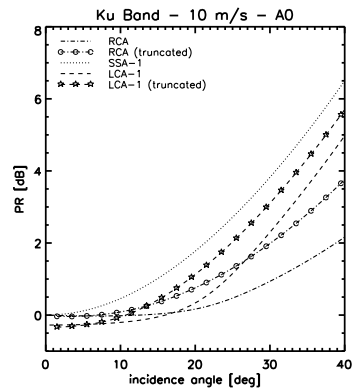

Figure 7. Left panel: NRCS versus incidence angle in VV polarization for a $10 \mathrm{~m} / \mathrm{s}$ wind speed in the case of an isotropic surface. Middle panel: Same in HH polarization. Right panel: PR versus incidence angle for a $10 \mathrm{~m} / \mathrm{s}$ wind speed in the case of an isotropic surface. Results are in Ku-Band and are predicted using RCA and LCA-1 coupled with a truncated (larger waves than $48 \times \lambda_{0}$ are removed) and a complete wave spectrum.

(a)

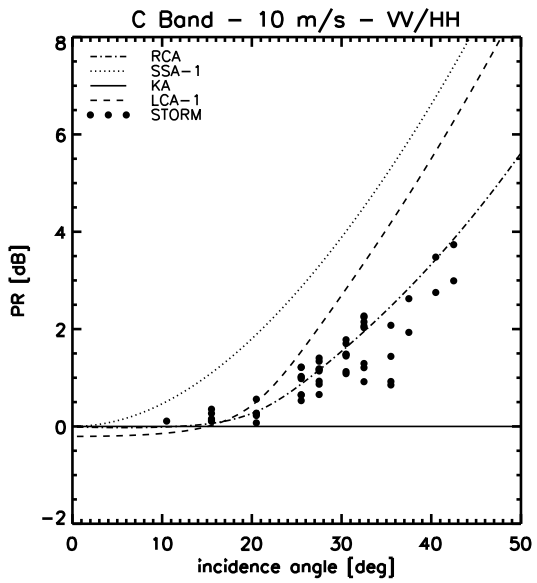

(b)

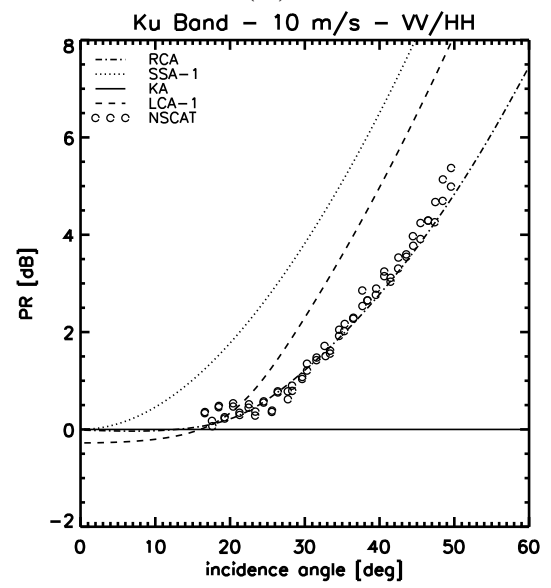

Figure 8. PR versus incidence angle for a $10 \mathrm{~m} / \mathrm{s}$ wind speed in the case of an isotropic surface. Results are in C-Band (left) and Ku-Band (right) and are predicted using KA, SSA-1, LCA-1 and RCA. C-Band data are from STORM radar. Ku-Band data are from NSCAT data. 
(a)

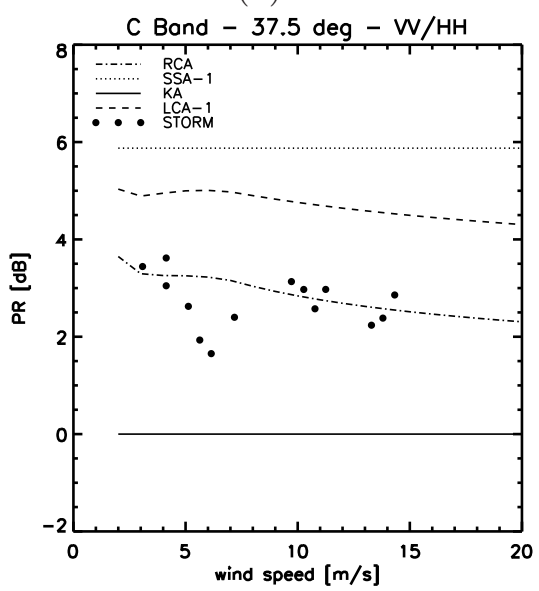

(b)

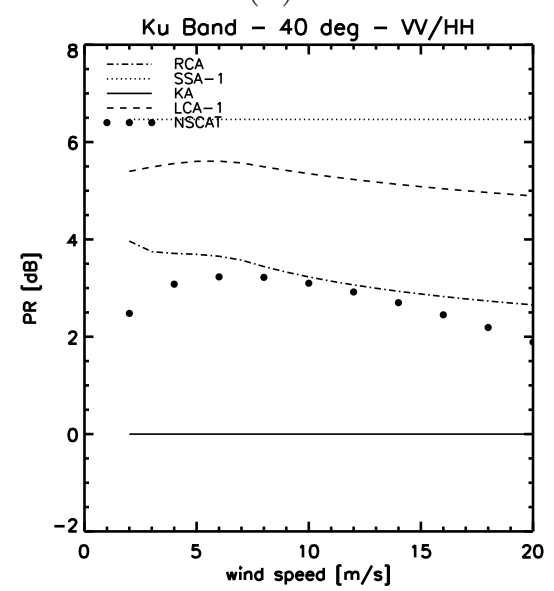

Figure 9. PR versus wind speed in the case of an isotropic surface. Results are in C-Band for a $37.5 \mathrm{deg}$ incidence angle (left) and $\mathrm{Ku}$-Band for a $40 \mathrm{deg}$ incidence angle (right) and are predicted using SPM-1, KA, TSM, SSA-1 and LCA-1. C-Band data are from STORM radar. Ku-Band data are from NSCAT data. 